\title{
Douleur Chronique, usages du corps et souci et soi
}

\author{
Dor crônica, usos do corpo, preocupação e cuidado \\ Chronic pain, body uses, care and concern
}

Valérie Bloch ${ }^{1}$

Resumo: Em uma era marcada pela robotização de empresas e serviços, a desmaterialização de documentos e a tirania de novas tecnologias, o aparecimento de uma nova categoria nosográfica repleta de a prioris, a de "dor crônica", me incitou a questionar as ligações do uso do corpo em um contexto de trabalho. Tomo como hipótese o entendimento que a dor crônica não é um sintoma das aforias contemporâneas em termos dos usos do corpo e, dessa forma, ela utrapassa amplamente o campo da medicina. A coleta de narrativas de vida entre profissionais da dança, tomados em sua habilidade de descrever o vivido permite revelar as dinâmicas de poder em jogo na utilização do corpo como uma ferramenta de trabalho. A forte credibilidade da fala desses sujeitos está ligada a uma profissão frequentemente tomada como "vocação". Essa perspectiva pode frustrar a tendência a relegar ao domínio da psiqué tudo aquilo que não pode ser provado em sua existência concreta. Uma aproximação genealógica e transversal dessas histórias de dor permite de identificar as "dobras" de resistência e recusa de toda medicação diante da dor e compreender como se chegou até esse ponto, entendendo que as práticas de cura são possíveis diante de uma alto preço que é a mudança de paradigmas.

Palavras-chave: dor, corpo. dança, dor crônica, poder, trabalho, ferimentos, práticas de cura.

Résumé : Dans une ère fortement marquée par la robotisation des entreprises et des services, la dématérialisation des documents et la tyrannie des nouvelles technologies, l'apparition d'une nouvelle catégorie nosographique «douleur chronique» entachée de beaucoup d'à priori

\footnotetext{
${ }^{1}$ Université de Montreal. E-mail: valdocfr@gmail.com.
}

Latitude, Vol. 9, nº 2, pp. 07-28, 2015

DOI: https://doi.org/10.28998/2179-5428.20150203 
Douleur Chronique, usages du corps et souci et soi

m'incite à questionner ses liens avec les usages du corps en contexte de travail. J'émets d'emblée l'hypothèse qu'elle n'est qu'un symptôme des apories contemporaines en termes d'usages du corps et dépasse ainsi largement le champ de la médecine. Le recueil de récits de vie auprès de professionnels de la danse, pressentis pour leur habileté à décrire leur ressenti me permet de révéler les dynamiques de pouvoir à l'œuvre au creux d'un corps outil de travail. La forte crédibilité de la parole reliée à un métier de "vocation" déjoue par ailleurs une tendance à reléguer dans le domaine de la psyché ce qui ne fait pas la preuve de son évidence. Une approche généalogique et transversale de ces histoires de douleur permet alors d'identifier les «plis» que sont l'endurance et le rejet de toute médication face à la douleur et comment on en est arrivé là, mais aussi que la guérison est possible au prix d'un changement de paradigme.

Mots-clés: douleur, corps, danse, chronique, pouvoir, travail, blessure, savoir, guérison.

\section{La douleur chronique}

Qui dit «douleur», dit souffrance: «Ça fait mal!» On pense à nos amis, à nos parents, à nous-mêmes. Quelle que soit la douleur, physique ou morale, elle suscite avant tout la compassion et réclame un soulagement rapide. Habituellement, elle est comprise comme un signal d'alarme, une invitation à suspendre son geste, un indice de désordre sous-jacent, mais quand elle persiste au-delà d'une certaine limite, fixée par les autorités médicales, elle devient un handicap et se voit qualifiée de chronique. Il peut s'agir de douleur du bas du dos, de lumbago, de sciatique, de douleur de la mâchoire ou encore de syndromes au nom savant: l'algodystrophie, le Syndrome Douloureux Régional Complexe ou la fibromyalgie. Le terme de douleur chronique est tout compte fait, un terme générique, qui désigne n'importe quelle douleur persistante de la migraine à la fibromyalgie, dès qu'elle dure de plusieurs mois au restant de vos jours (Suddath, 2011, p.74) $)^{2}$.

2 «Chronic pain is an umbrella term for any type of persistant pain, from migraines to fibromyalgia, that lasts anywhere from several months to the rest of your life» 
Pour Isabelle Baszanger, spécialiste en la matière, ce sont les progrès de la science en matière d'anesthésie qui, couplés à un glissement socioprofessionnel autour de progrès technologiques, ont présidé à l'apparition d'une nouvelle maladie et d'un nouveau discours (1990). C'est en effet le Dr John J. Bonica, anesthésiste de formation, qui ouvre la première clinique de la douleur en 1961 à Seattle pour répondre aux besoins identifiés à l'hôpital de Tacoma, dans l'état de Washington, auprès des blessés de retour du front du Pacifique de 1944 à 1946 et aux prises avec des syndromes douloureux prolongés. Parce que le corps médical est désigné pour soulager les souffrances morales autant que physiques, surtout depuis le désintérêt pour le religieux, c'est à lui qu'incombe le fardeau du contrôle de la douleur. Paradoxalement, c'est donc sur la vocation compassionnelle de la médecine que s'est construite cette nouvelle catégorie nosographique "douleur chronique» à une époque où les produits anesthésiques sont de plus en plus performants.

Mais curieusement, l'accès à des médicaments efficaces semble dispenser du colloque singulier avec un patient souffrant. Qui plus est, en supprimant le symptôme et en masquant probablement le problème sousjacent, les traitements donnent alors l'illusion du soin et conduisent inévitablement à l'escalade des moyens, parfois même à la dépendance aux médicaments. Ils entretiennent par la même occasion l'idée d'une maladie incurable et mystérieuse, voire imaginaire. Dans les discours, il est souvent question de déviance, de nouvelle forme d'hystérie, de bénéfices secondaires, d'imposture, de carrière, très peu de souffrance humaine. Pourtant si on y regarde à deux fois, cette nouvelle catégorie de maladie, baptisée «douleur chronique», désigne avant tout un phénomène aux contours imprécis qui rend plutôt compte de l'inadéquation du modèle clinique qui veut objectiver une lésion pour l'inscrire dans un régime de vérité.

«Une deuxième source de confusion vient de tentatives infructueuses de combiner expérience et sensation dans le modèle biomédical de base. Tandis que beaucoup de chercheurs sur la douleur acquiescent avec la définition proposée par le Souscomité de Taxonomie de l'Association pour l'étude de la Douleur qui considère que la douleur « est une sensation désagréable et une expérience émotive 
Douleur Chronique, usages du corps et souci et soi

associée à un dommage tissulaire actuel ou potentiel ou décrit comme tel» (Jackson, 2005, p. $337)^{3}$.

Une fois désignée par le monde médical comme une nouvelle catégorie nosographique, il importe de situer la douleur chronique en regard de la culture de médecine dont elle est issue et de son histoire pour mieux en comprendre les tenants et les aboutissants.

Jusqu'au cinquième siècle avant J.C., la médecine, dite grecque, s'inspirait de la mythologie et comprenait la douleur comme un signe des dieux, qui nécessitait prières et offrandes auprès de la déesse Panacée pour obtenir sa guérison. C'est Hippocrate (île de Cos 460-Larissa, Thessalie, vers 377 avant J.-C.), dont notre système médical revendique la filiation, qui impose de rompre avec toute croyance magico-religieuse, mais c'est seulement au siècle des Lumières, que l'objectivation de la maladie devient une condition sine qua non de prise en charge thérapeutique. Faisant suite à une approche théorique du corps humain, basée sur les «humeurs», concept abstrait s'il en est, les dissections d'Ambroise Paré établissent un lien de cause à effet entre lésions anatomiques visibles et symptômes énoncés. Dès lors, la médecine réussit à superposer, «l'espace de configuration de la maladie et l'espace de localisation du mal dans le corps» grâce aux «privilèges accordés à l'anatomie pathologique» (Foucault, 1963, p.1). C'est ce moment de surgissement d'une médecine positiviste, où ce que l'on voit, «l'évidence», prime sur ce que le malade ressent, «le subjectif», que Michel Foucault désigne comme celui de la «naissance de la clinique» (1963). Le souci d'objectivité, qui se réclame d'un esprit résolument scientifique, de plus en plus classificateur et méthodique, devient alors prioritaire. Se voulant ainsi basée sur des évidences, la médecine délaisse sa mission première, le soin, et se laisse subrepticement envahir par les mêmes exigences de résultats que le monde des affaires (Halioua, 2009).

\footnotetext{
3 «A second source of confusion derives from unsuccessful attempts to incorporate experience and feeling into a basically biomedical model. Whereas many pain researchers agree with the definition supplied by the International Association for the study of Pain Subcommittee on Taxonomy that pain is «an unpleasant sensory and emotional experience associated with actual or potential tissue damage, or described in terms of such damage»»
} 
La douleur en elle-même n'est donc pas un phénomène nouveau comme en témoignent la plupart des ouvrages médicaux depuis l'Antiquité (Rey, 1993). C'est plutôt sa qualité éminemment subjective qui vient nouvellement confronter l'impératif d'objectivité que s'est donné une médecine devenue positiviste. Ce qui change donc ce n'est pas la douleur, mais plutôt le regard médical, qui devient de plus en plus obsessif, au détriment d'une écoute sensible de l'histoire de la maladie et de la part singulière invisible de la souffrance humaine.

«Les figures de la douleur ne sont pas conjurées au bénéfice d'une connaissance neutralisée; elles ont été redistribuées dans l'espace où se croisent les corps et les regards. Ce qui a changé, c'est la configuration sourde où le langage prend appui, le rapport de situation et de posture entre ce qui parle et ce dont on parle» (Foucault, 1963, p.VII).

L'obsession de voir les lésions pour croire le patient n'a cessé de faire le jeu des nouvelles technologies. C'est ce regard scrutateur qui se prolonge par l'échographie, la radiologie, la cœlioscopie, la laparoscopie, et toutes les techniques invasives qui n'ont d'autre objet que de voir et d'authentifier une lésion qui viendrait objectiver la maladie et prouver la véracité des dires du patient. Le regard médical posé sur le corps l'inscrit alors dans un contexte politique et participe de ce que Michel Foucault appelle la technologie politique du corps:

«On y traiterait du corps politique comme ensemble des éléments matériels et des techniques qui servent d'armes de relais de voies de communication et de points d'appui aux relations de pouvoir et de savoir qui investissent les corps humains et les assujettissent en en faisant des objets de savoir» (Foucault, 1975, p. 33).

De ce point de vue, il apparaît clairement que la douleur chronique, par sa résistance au traitement et son irréductibilité, met dangereusement en péril l'autorité médicale. Elle révèle ainsi une des failles de son savoir 
Douleur Chronique, usages du corps et souci et soi

basé sur des évidences et sur l'objectivation des signes et questionne toute une culture de médecine, détournée de son sens premier, le soin, vers des exigences de résultats et des certitudes d'ordre statistique. J'émets alors l'hypothèse que la douleur chronique s'organise selon le modèle d'un dispositif ${ }^{4}$ témoin de la multiplicité des dynamiques de pouvoir à l'œuvre. Le corps devient alors le lieu d'observation privilégié des différents enjeux sociaux contemporains qui $s^{\prime} y$ croisent et $s^{\prime} y$ entrecroisent et qui se déclinent selon des lignes de discours et de spatialisation, de force et de fracture auxquelles j'ajoute des lignes de subjectivation.

«Démêler les lignes d'un dispositif, dans chaque cas, c'est dresser une carte, cartographier, arpenter des terres inconnues et c'est ce qu'il appelle le «travail sur le terrain». Il faut s'installer sur les lignes mêmes qui ne se contentent pas de composer un dispositif, mais qui le traversent et l'entraînent, du nord au sud, d'est en ouest ou en diagonale. »(Deleuze, 1989, p. 185).

Cette nouvelle catégorie nosographique pose ainsi la question des usages du corps dans une société de consommation basée sur le profit et ramène au premier plan un corps désormais singulier, inapte au travail, avec des limitations et des dysfonctions, et qui, de fait, se situe en dehors de la norme. En témoignant d'un corps souffrant, inapte à la production, la douleur chronique dit tout haut la souffrance du travailleur ou du malaimé tout en menaçant l'équilibre social. Le corps devenu non-productif entraîne en effet toute une série de mesures disciplinaires et de réactions en chaîne, qui témoignent des relations de pouvoir à l'œuvre. Car, même si

4 «Par dispositif, j'entends une sorte - disons - de formation qui, à un moment donné, a eu pour fonction majeure de répondre à une urgence. Le dispositif a donc une fonction stratégique dominante... Le dispositif donc est toujours inscrit dans un jeu de pouvoir, mais toujours lié aussi à une ou à des bornes de savoir qui en naissent mais tout autant le conditionnent. C'est ça le dispositif: des stratégies de rapport de force supportant des types de savoir et supportés par eux.» (Dits et écrits, vol.III, p.299)» (Foucault, dans Agamben, 2007: $10)$. 
elle est difficilement objectivable, la douleur chronique a des effets bien visibles et quantifiables. Les coûts qui s'y rattachent, en particulier en cas d'indemnisation après un accident du travail, la rendent bien réelle et en font un problème de société toujours irrésolu qui réclame l'attention. Ainsi, même si la douleur chronique nous renvoie à notre dimension sensible et nous touche, elle interroge aussi la double contrainte qui nous lie à l'État Providence.

Parce qu'elle survient dans un moment critique où les vieux modèles peinent à l'inclure dans leur cadre trop étroit, j'émets l'hypothèse que la douleur chronique est le reflet des apories contemporaines en termes d'usages du corps et de rapports de productivité et qu'elle rend compte d'une double détresse, à la fois sociale et existentielle. Elle pose la question du rapport à l'altérité et au changement, des multiples façons de mater les révoltes ou de nier les problèmes. De fait, elle interroge les limites du paradigme mécaniste, de l'universalité des corps, de l'hégémonie du matériel sur le spirituel, d'un régime économique basé sur la richesse issue de la force de travail.

Pour Arthur Kleinman, en touchant davantage les classes sociales les moins éduquées et donc, les plus sujettes à exercer des travaux lourds, la douleur chronique rendrait plus évidentes les lignes de fracture sociale (Kleinman, 1992). J'ai souhaité vérifier cette affirmation en interrogeant les caractéristiques d'une douleur qui touche aussi un milieu habituellement considéré comme éduqué et riche d'un fort capital symbolique, celui de la danse. En choisissant une catégorie socio-professionnelle, celle des professionnels de la danse plutôt qu'un type de douleur spécifique, je souhaite interroger la relation au travail et au corps plutôt que la relation à la santé et à un type de douleur particulier. J'observe donc les douleurs en lien avec les usages d'un corps-outil de travail, mais aussi corps du sujet, tout désigné pour rendre compte des conflits à l'œuvre au creux de cet «outil du danseur, le corps, qui, parce qu'il se confond avec l'être (un violoniste n'est pas son violon), finit par envahir toute la danse» (Verrièle dans Bastin, 2010).

J'émets alors l'hypothèse qu'au-delà de la lutte de classes, la douleur chronique nous renseigne sur les luttes de pouvoir «intestines» qui traversent le corps du sujet autant que le corps social au creux d'une société de plus en plus mécanisée et pourtant de plus en plus confrontée à un corps sensible avec ses limites et ses lois intrinsèques. Tandis que la 
Douleur Chronique, usages du corps et souci et soi

douleur chronique d'un manutentionnaire ou d'un chauffeur d'autobus questionne plus directement les conditions de travail et valide plus aisément l'hypothèse de la simulation, comme moyen d'échapper à une charge de travail excessive, celle du danseur professionnel bénéficie de sa réputation d'exercer un «métier de vocation» qui rend la plainte d'emblée légitime (Sorignet, 2004): «Pour moi, la danse c'est tellement ma vie! que tout d'un coup, quand ça c'est arrivé, je me suis dit: «Mais, qu'est ce que je vais devenir, si je peux plus danser? Je suis qui? Je suis quoi?»» (Lola).

En choisissant d'examiner ce phénomène, «douleur chronique», sous l'angle du corps et des usages du corps, d'emblée, j'envisage les usages du corps, comme «des techniques et l'ouvrage de la raison pratique là où on ne voit d'ordinaire que l'âme et ses facultés de répétition» (Mauss, 1934, p.7). J'adopte ainsi la vision de «la nature sociale de l'habitus», que Marcel Mauss définit comme ces «habitudes» qui «varient non pas simplement avec les individus et leurs imitations», mais «varient surtout avec les sociétés, les éducations, les convenances et les modes, les prestiges» (Mauss, 1934, p. 7). Par son approche sur les techniques du corps, Marcel Mauss se fraye un chemin entre toutes les disciplines qui s'intéressent au corps, pour y affirmer un point de vue à la fois unique, central et englobant, celui d'un corps perçu comme donnée universelle et marqueur historique des usages sociétaux en cours (Mauss, 1934). Pour lui, la course, la nage et même la marche, ne sont pas seulement des pratiques, qui varient d'une époque ou d'une société à l'autre. Ces «techniques» en disent long sur l'éducation reçue, les modèles copiés, les habitudes de vie et les stéréotypes, mais aussi sur les forces à l'œuvre dans l'apprentissage d'un métier. Ainsi, les usages du corps dans le monde de la danse au Québec, envisagés avec le même «triple point de vue, celui de l'homme total, qui est nécessaire» rendent compte à la fois des données physiques, anatomiques ou physiologiques et phénoménologiques, mais aussi sociales et politiques (Mauss, 1936). Le corps y est compris comme le premier marqueur de notre évolution en termes d'espèce, de notre identité d'individu unique d'autant que la danse est un des modes d'expression et de socialisation les plus populaires et les plus partagés.

\section{Le Contexte}

Historiquement donc, la danse témoigne d'une double évolution, 
celle des usages du corps et des paradigmes sociétaux. Ainsi, même si danse moderne et contemporaine s'inscrivent également en faux en regard des contraintes imposées par le ballet classique, elles reflètent chacune à leur façon les crises majeures que traverse le corps social. La danse d'Isadora Duncan, contemporaine de la crise de 1929, privilégie un style libre de toute contrainte et en cela, s'inscrit parfaitement dans le mouvement «d'émancipation de jeunes femmes qui réagissent au puritanisme et à la place qui leur est faite dans la société» en refusant tout particulièrement de porter un corset (Izrine, 2002, p. 31). À sa suite, Martha Graham inscrit encore un peu plus la danse moderne américaine dans un mouvement d'émancipation des femmes en faisant «dépendre la force du geste de la force de l'émotion» et en situant «le centre de gravité du mouvement dans la ceinture pelvienne», plutôt que dans le haut du corps (Izrine, 2002, p.42). Merce Cunningham, quant à lui, commence à «inscrire l'idéal de rectitude qui érige la pensée occidentale du corps comme progrès de la raison sur l'émotion ou sur la pulsion dans le corps des danseurs» dès 1945, juste après la seconde guerre mondiale (Izrine, 2002, p.78). Issue d'un milieu intellectuel et universitaire, très proche de l'expressionnisme abstrait américain, sa danse inscrit le corps dans un jeu de constructiondéconstruction et de hasard, très inspiré de la musique de John Cage, de ses silences, de son choix de rythmes, de notes aléatoires et de son piano préparé.

En Europe, Pina Bausch témoigne à sa façon du traumatisme de la seconde guerre mondiale, de l'impossibilité de revendiquer une identité allemande, de faire appel à la mémoire ou de s'inspirer du courant expressionniste et de sa gestuelle, sous peine de s'associer immanquablement au régime nazi dans sa toute première chorégraphie, im Wind der Zeit, en 1969 (Izrine, 2002). L'Allemagne d'après-guerre se retrouve en effet coupée en deux et coincée entre deux blocs, l'Amérique à l'ouest, et la Russie à l'est. Le style de Pina Bausch témoigne alors de cette rupture qui force un renouvellement radical de toute forme d'expression et impose un cadre très serré à la création.

$\mathrm{Au}$ Québec, la danse contemporaine s'inscrit dans une sorte de «tradition de non-tradition» et en se qualifiant de «Nouvelle danse», non seulement elle refuse toute filiation, mais elle fait de l'isolement un marqueur identitaire, une preuve de son originalité et bientôt une marque de commerce (Tembeck, 2002). Françoise Sullivan et Jeanne Renaud, toutes 
Douleur Chronique, usages du corps et souci et soi

deux signataires du manifeste du Refus global, initié par le peintre PaulÉmile Borduas en 1948 et que d'aucuns relient au mouvement automatiste d'André Breton et au surréalisme, se sont pourtant formées à New York auprès d'élèves de Martha Graham. Mais ce refus de tradition est peut-être à comprendre en regard de l'affirmation que la danse contemporaine n'est pas issue de la danse.

"C'est Cunningham qui a inventé, à partir de la danse un art qui s'appellerait: la danse. À partir de cette activité élémentaire, familière aux enfants et aux fous, qui nous libère des fonctions ordinaires, nous procure l'heureuse illusion de n'entretenir que des rapports linguistiques avec le temps et l'espace, nous soumet tout entiers aux décisions de notre corps c'est-à-dire: nous libère d'un sens extérieur à lui» (Louppe, 1988, p. 7).

L'histoire de la danse contemporaine retrace donc une certaine filiation même si c'est sous la forme de ruptures ou de discontinuité.

«Car une danse faite de variations infinies sur un vocabulaire gestuel, pure déclinaison de paradigmes, étouffe (comme on a pu le voir avec certains chorégraphes américains). Et elle n'a comme issue que la fuite en avant, toujours plus d'énergie et de virtuosité» (Durand, 1988, p. 35).

Mais là comme ailleurs, il est désormais question de productivité et de former des «professionnels» du métier: «En d'autres termes, c'est sur un modèle académique, disciplinaire et soucieux de rentabilité que se pense encore aujourd'hui la formation professionnelle à la pratique contemporaine en danse» (Ginot et Launay, 2002, p. 107). Aujourd'hui, le milieu de la danse québécoise publie son plan directeur pour les dix prochaines années. On y retrouve les idées maîtresses de toute entreprise culturelle soucieuse de rassurer les investisseurs institutionnels et de garantir la qualité du produit culturel. Les valeurs mises de l'avant sont toujours l'innovation, la spécificité du produit québécois et son rayonnement à l'étranger. 
«Ce plan dévoile, de façon exhaustive et concrète, la vision et les stratégies de déploiement d'un domaine disciplinaire reconnu pour sa créativité, son dynamisme et son rayonnement» (Yvan Gauthier, PDG du conseil des arts du Québec) (Plan directeur de la danse professionnelle au Québec 2011-2021).

L'accent est mis sur l'organisation, l'attribution de moyens matériels et financiers et une formation de qualité, autant dire académique. La création y est appelée de ses vœux et la recherche souhaitée, mais nullement soutenue. Enfin, la rencontre d'un public de plus en plus large laisse entrevoir le lien entre financement et valeur marchande. Rien à voir avec le soutien d'une activité artistique, mais plutôt avec celle d'un «divertissement», apprécié à la taille de son public.

«C'est indubitablement un milieu énergique, stimulant, créateur et innovateur. Des qualités indispensables dans une société qui doit compter de plus en plus sur ses capacités innovantes et créatrices pour croître et se développer. La danse est certainement un atout important pour notre culture, voire notre économie» (Louise Roy, présidente du conseil des arts de Montréal) (Plan directeur de la danse professionnelle au Québec 2011-2021).

Mais de l'avis général, les danseurs sont épuisés, éprouvés par plusieurs coupes budgétaires et devenus dépendants des subventions. De ce fait, ils acceptent les principes de bonne gouvernance, dictés par les décideurs, confiants qu'en se soumettant à l'autorité et aux principes gestionnaires et comptables, ils se verront soutenus à l'instar des pays en voie de développement soumis aux lois du FMI, en échange d'aide financière au développement.

Parce qu'elle surgit au lendemain de l'ère industrielle, quand la mécanisation du travail met le corps en concurrence avec la machine, la douleur chronique vient tout particulièrement questionner les usages du 
Douleur Chronique, usages du corps et souci et soi

corps contemporain dans un monde axé sur la performance et la productivité.

«La société, asteure, elle roule à 100 milles à l'heure, puis t'as pas le temps de $t^{\prime}$ occuper de toi [...] faut que tu coures aller chercher tes enfants, faut tu coures faire ci, faire ça... Mais s'ils prenaient le temps un peu de s'en occuper, bien, regarde, ça irait déjà pas mal mieux. Parce que les maux de dos puis les burn out, puis les dépressions, m'a te dire que... moi, tout le monde connaît quelqu'un qui a eu ça.» (Participant 11, temps final) (Baril, 2008 p. 47).

À cet égard, le territoire nord-américain se fait particulièrement remarquer au début du vingtième siècle par son culte du corps, son goût pour le jogging, l'aérobic et le bodybuilding. Antonio Gramsci, quant à lui, souligne dans ses Carnets de Prison (1929-1935) combien le Protestantisme y a favorisé l'implantation de méthodes de travail propres au Taylorisme et au Fordisme. Cette influence y est d'autant plus remarquable qu'elle repose sur des principes d'autodiscipline et qu'elle s'exerce de façon subtile au lieu de s'imposer comme force idéologique (Turner, 1984). Le corps, en particulier, y est souvent perçu, comme un outil, un objet soumis au bon vouloir du sujet et aux stéréotypes du moment. Cette tendance s'accentue en milieu québécois francophone, en contexte de lutte de classes, de conflits identitaires, de conflit de croyances et de luttes d'influences entre idéologie catholique et protestante: «Les personnes dépressives, démoralisées ou indolentes sont alors considérées comme des êtres socialement invalides en regard d'un «prestige de l'énergie» (Devereux 1980)» (Massé, 1999: 53). Pourtant, outre les douleurs de courbatures, le métier de danseur ne paraît pas d'emblée très exposé aux douleurs chroniques. La croyance populaire veut d'ailleurs que les danseurs prennent particulièrement soin de leur corps puisque, sur scène, ils présentent un corps «idéal», parfaitement sain, agile et performant. C'est en cela qu'un regard porté sur les «coulisses» d'un corps idéalisé, et pourtant soumis aux lois de l'usure et aux risques d'accident, me permet de questionner cette évidence. Car les blessures y sont nombreuses et les douleurs chroniques aussi. Le corps dansant est aussi un corps souffrant, parfois en conflit de travail et aux prises avec des questionnements à l'autorité, autorité de la douleur sur le geste comme du chorégraphe sur l'interprète, au sein d'un corps social bien particulier: le corps de la danse. 
En orientant le terrain de ma recherche sur le corps et ses usages, comme en un lieu où se côtoient toutes les disciplines, je rejoins Marcel Mauss, mais en y observant le ressenti, j’en fais aussi un lieu de vérité où la douleur fait corps avec le sujet: «Le corps commence [...] au point où commence la douleur» (Artaud, dans Rogozinski, 2011). Le recueil de récits de vie s'impose alors comme la méthode de choix pour aborder la temporalité et le vécu caractéristiques de toute souffrance et de toute expérience subjective de la maladie: "Although the experience of pain is difficult to express in language, in their narratives my participants integrate the pain into their lives ${ }^{5}$ (Becker, 1999, p. 73). Qui plus est, si la douleur aiguë échappe à toute tentative de symbolisation, la douleur chronique se prête davantage à une construction historique et donne lieu à l'élaboration d'un discours sur le monde de la douleur. Ainsi, la prise en compte du micro-contexte d'un vécu quotidien avec la douleur devient essentielle et le récit de vie prend toute sa place dans une recherche d'accès au sens (Kleinman, 1992: 172). Je prends ainsi les dires pour acquis, comme le fait Jacob Rogozinski dans sa relecture de l'œuvre d'Antonin Artaud où il apparaît clairement que l'auteur du «pèse-nerf» présentait tous les symptômes d'une fibromyalgie, cependant qu'il décrit la douleur comme de tous les affects, le plus intense, «l'affect de la limite corporelle», celui qui «fait peau» et qui renvoie le plus à la chair, tandis que «la vérité première, la seule vérité qui tienne est que je suis et elle se révèle dans l'absolue certitude de ce que je sens [...]» (2011, p.129)

\section{Les données}

En les récits côte à côte, les dynamiques qui favorisent l'apparition d'une blessure et celles qui contribuent à leur guérison se dégagent. Chacun des treize récits révèle alors clairement que la douleur est tout sauf imaginaire et qu'elle rend compte d'une blessure profonde en lien avec les usages du corps et l'accumulation de leurs effets dans le temps et dans l'espace. Il y est question d'usure, d'érosion des articulations, d'arthrose sévère, de hernies récidivantes, mais malgré la diversité des dénominations et des dynamiques à l'œuvre, il en ressort que toutes ces expériences

5 «Même si l'expérience de la douleur est difficile à mettre en mots, dans leurs récits de vie, mes participants l'intègrent dans leur vie». 
Douleur Chronique, usages du corps et souci et soi

douloureuses relèvent d'un même champ, d'un même domaine, celui du ressenti et du vécu.

\section{La généalogie ou comment on en est arrivés là}

Avec la distance et le recul procurés par le récit, tous les interprètes livrent une analyse rétrospective assez précise de la façon dont s'est produite la blessure et comment elle donne lieu à une douleur durable. Ils et elles soulignent l'impact négatif de la répétition d'un même geste ou d'une même situation perçue comme déstabilisante et comment cela mène à une rupture d'équilibre ou à un blocage. La répétition du choc, si petit soit-il, et son étalement dans le temps sont des facteurs d'élaboration de blessures récurrents dans presque tous les récits, à deux exceptions près quand la rupture d'équilibre est précipitée par l'intervention d'une tierce personne: les blessures de Denyse et de Jeanne ont découlé d'une manipulation externe inappropriée. Bien qu'il s'agisse ici de thérapeute corporel ou d'enseignant de yoga, et non de médecins, les gestes qu'ils ont porté sans concertation préalable témoignent aussi d'un déséquilibre dans la relation, engendré par l'autorité conférée au savoir expert auquel elles ont toutes deux confié un corps «docile» (Foucault, 1975). Ainsi, la douleur, la blessure, la maladie et la mort résultent parfois de gestes, dits «iatrogènes», posés sans tenir compte des dires du sujet, de son avis, de son ressenti, ni de sa connaissance intrinsèque. Bien sûr, dans l'un et l'autre cas, il s'est agi d'un petit détail qui est venu faire basculer un corps déjà en équilibre instable: Jeanne et Denyse avaient sans doute une fragilité passée inaperçue quand l'accident est survenu. Peut-être aussi que l'historique des usages du corps n'a pas suffisamment été pris en compte même si Jeanne fait référence à une blessure de jeunesse dont elle ne s'est souvenue qu'à posteriori et comme pour excuser son chiropracteur.

Béatrice, quant à elle, évoque une lésion qui s'est «build, build, build $»^{6}$, Patricia, une succession de microtraumatismes: «Je pense que ça s'est installé. J'ai dû avoir une succession de chocs...» Elle nomme aussi plusieurs évènements qui ont contribué à créer les conditions de la blessure: sa grossesse, qui a eu des effets sur son corps et sa vision du monde, en même temps que sa participation à la syndicalisation des

${ }^{6}$ Accumulée, accumulée, accumulée 
danseurs, qui lui a révélé les «conditions absurdes» de travail des interprètes.

Jeanne se représente les effets de la danse sur son corps comme des micro-blessures, qu'elle juge pourtant nécessaires. Caroline énonce aussi que sa blessure au dos s'est construite dans le temps, sur un déséquilibre initial: «Moi, je pense que ça a été un déséquilibre graduel, qui s'est fait après ma chute sur le coccyx.» Dans son cas, comme dans celui de Jeanne, la survenue de la blessure ramène à la mémoire des accidents oubliés, des blessures de jeunesse qui, même si elles sont survenues en dehors de tout contexte professionnel, ont laissé des traces et sont restées inscrites dans la mémoire corporelle.

Sylvie et Caroline insistent toutes deux sur les facteurs de risque et les circonstances favorisantes: l'influence du stress et de l'environnement professionnel, l'ambiance de travail. Caroline invoque plus particulièrement une sorte d'état émotionnel, qui déconnecte le corps et l'esprit, ce qu'elle appelle le body-mind, et qui lui ferait perdre le contrôle de son corps. Sylvie met surtout l'accent sur l'effet pervers des émotions qui mettent le corps à risque de se blesser. Elle reconnaît sa grande sensibilité aux ambiances de travail et à la qualité des relations professionnelles: «Just get stressed in my head, I get stressed all the way through my body... ${ }^{7}$ [Rit]» Elle nous donne une description subtile de la dynamique qui lie le corps et l'esprit, là où le terme esprit recouvre aussi bien la pensée que l'affectif et le domaine des émotions:

«I think I didn't realise that until I hurt my ankle, cause that was a definite point where I knew I was angry, and I knew I shouldn't have done what I did cause, I pushed my body with that anger rather than with the technic that pushes you into a jump; I was doing that out of anger. When I looked back on that, I realised that: «OK, don't jump in that sort of way again! It's taking something out of your body that's not your body's fault...» ${ }^{8}$ (Sylvie).

\footnotetext{
7 «Il suffit que je sois stressée dans la tête pour être stressée tout au long de mon corps.»

8 «Je pense que je ne l'avais pas réalisé jusqu'à ce que je me foule la cheville, parce qu'à ce moment-là, je savais que j'étais en colère et je savais que je n'aurais pas dû faire ce que j'ai fait parce que j'ai poussé mon corps avec cette
} 
Douleur Chronique, usages du corps et souci et soi

Les différents enjeux liés à la carrière, à la difficulté de la performance ou à l'approbation du public, des pairs, des critiques ou des chorégraphes, sont bien des causes de stress et de tensions qui fragilisent aussi l'interprète. C'est le propre des danseurs et des danseuses, ayant une grande conscience de leur corps que d'être très sensibles à leur environnement, en particulier affectif et émotionnel. C'est une question d'équilibre fondamental, d'équilibre de l'être qui conditionne l'équilibre tout court. Renée en est persuadée:

«À chaque fois que j'ai eu des accidents, c'était toujours parce qu'il y avait quelque chose d'émotif là-dedans. On [ne] peut pas, et mon ostéopathe me le dit, là! On est obligés de chercher un équilibre parfait pour danser. On $[n e]$ peut pas, émotionnellement, venir nous déstabiliser avant de danser! On va se blesser!»

Le corps dansant a besoin de toute sa concentration pour "performer» sans danger. Il est question d'état d'esprit et de ses effets sur le corps et ses usages, mais aussi d'agressions verbales et d'émotions perturbatrices qui sortent le corps de son axe et le déséquilibrent en changeant les points d'appui du corps et de l'esprit et les dissocient. Une seconde d'inattention et c'est la chute, l'accident, l'entorse, la hernie ou la déchirure. Le corps n'est pas une machine et il n'est pas insensible à ce qui lui arrive. Le corps a des limites, qu'il ne peut repousser indéfiniment. Qui plus est, il est doué de mémoire et est sujet, lui aussi, au surmenage. S'il suffit donc de très peu de choses pour déséquilibrer le danseur, de bonnes conditions de travail, dans un climat de saine communication et une attention à soi permanente s'avèrent les meilleurs garants de la santé et de la sécurité au travail.

colère plutôt qu'avec la technique qui te propulse dans un saut; je faisais ça par colère. Rétrospectivement, je me suis dit : Bon, ne saute plus de cette façon! Ça fait porter la responsabilité à ton corps, alors qu'il n'y est pour rien». 


\section{L'endurance $=$ un trait culturel}

L'endurance s'inscrit dans tous les récits selon une ligne de subjectivation, témoin d'une posture proche d'une déformation professionnelle ou d'une culture de métier ${ }^{9}$ qui traverse toutes les pratiques, de la plus classique à la plus contemporaine. Ce trait reflète à la fois une attitude physique et mentale face à la douleur ainsi qu'une loi implicite, très répandue dans le monde des arts et du spectacle. Cette loi s'apparente à celle du silence, du maintien des apparences, et rejoint ainsi les lignes d'énoncé et de visibilité du dispositif selon lequel se construit la douleur chronique. Ainsi Jeanne n'a rien dit pendant douze années consécutives, même à sa meilleure amie. Son silence participe pleinement de sa volonté de persévérer envers et contre la douleur. Quant à Denyse, il est encore difficile aujourd'hui de recueillir ses confidences en matière de douleur. Elle préfère de loin parler d'une guérison qu'elle attribue d'ailleurs à la force de sa volonté, à son leadership et à sa capacité à décider. Si l'endurance est particulièrement encouragée dans le milieu, elle reflète aussi une aspiration constante au dépassement de soi, partagée par toutes et tous. Dans le monde de la danse, il est question de rapport aux pairs, aux collègues et au public, mais aussi d'idéal du moi, de plan de carrière et s'agissant du corps, de fantasme de toute puissance. Le rapport à la douleur prend appui sur des lignes de force de nature professionnelle qui semblent avoir pénétré subrepticement la dynamique personnelle et y font écho:

«Je vais te dire y a plein de fois où j'ai dit à quelqu'un: «Prends soin de toi!» à un gars là...Qu'est ce que j'en ai à foutre qu'un gars y s'arrête ou pas... Je [ne] vais pas prendre sa place... Donc c'est comment nous on le vit, comment nous on [ne] veut pas laisser la place parce que on sait que ça passe vite là... (Ça passe vite? Tu veux dire...) Ça veut dire que... On sait que notre corps ça va être notre limite et que si on est blessée on [ne] va

$9 \quad$ Le terme de culture renvoie ici à une forme de construction collective qui traverse des institutions qui agissent comme des systèmes qui se renforcent. 
Douleur Chronique, usages du corps et souci et soi

pas faire tel ou tel spectacle et puis on veut faire tous les spectacles, on veut danser...On n'est pas là pour notre salaire... Notre salaire c'est la scène... (Et vous savez que votre corps c'est la limite?) Oui. (Tous?) Ben oui, on sait que c'est la limite, mais on se croit des demi-dieux... Et puis quand on a de la douleur on se dit ben, c'est moi qui décide de la limite... Je peux endurer» (Renée).

«Mais c'est dans cette période-là que c'était vraiment, je dirai, au-dessus de..., de l'entendement. Quand j'ai vécu ça, en fait c'est simple, le corps c'est une dynamique, mais l'esprit... L'esprit voulait tellement... Que le corps a abdiqué!» (Louis)

Ainsi les professionnels de la danse développent une stratégie défensive d'ordre social, puisqu' associée à leur communauté d'appartenance professionnelle et inscrite dans une culture de l'endurance, proche de celle décrite chez les professionnels de la santé aux prises avec une réalité qui exploite leur passion ou leur dévouement (Maranda et al., 2006).

«On est en train de jouer sur un mode où il faut être complètement dévoué ... Un blessé de guerre là, il est sur le champ de bataille là, pis faut continuer à tirer pis à lancer pis donner sa vie, c'est ça la vie de danseur... Si t'es blessée là, mais qu'on te dit Non! [tu ne peux pas t'arrêter!] Mais il faut quand même [continuer de danser]... C'est comme un soldat, il faut qu'il continue, c'est une question de vie ou de mort dans le sens que, c'est ça on va jusqu'à la mort...(Ah oui? C'est implicite?) Oui, mais parce que, on nous l'inculque, mais aussi parce que c'est une passion. $C^{\prime}$ est comme les gens qui s'aiment passionnément. $C^{\prime}$ est à la vie à la mort et donc c'est facile de jouer là-dessus. C'est trop facile et ça je suis énormément fâchée, que eux ne comprennent pas, qu'ils ne 
croient pas en notre dévouement sincère et que quand nous on dit qu'on est blessée, c'est qu'on est blessée. $\mathrm{Y}$ en a peut-être un ou deux fainéants qui [ne] veulent juste pas travailler un jour ou un autre...» (Renée, salariée d'une grande compagnie).

De toute évidence, l'endurance participe de la chronicité en favorisant le maintien des usages du corps, tout en résistant le plus possible à l'appel de la douleur. Il faut donc réintroduire de la durée et de la temporalité dans la compréhension de la chronicité de la douleur et de son lien de causalité avec la capacité d'endurance, forme de résistance à la douleur et, de fait, au traitement précoce de la blessure initiale. Dans tous les cas, il apparaît clairement que, plus l'endurance est élevée, plus les chances de chronicité sont grandes.

L'endurance a des limites, le seuil de la douleur, qui mesure plutôt la capacité à endurer la douleur, que son intensité à proprement parler, et que les récits de vie situent unanimement toujours très au-dessus de la moyenne. Mais l'endurance se manifeste aussi par un rejet de toute médication, une relation pour le moins distanciée de la médecine et enfin un désir effréné de continuer à danser malgré la douleur.

\section{Un seuil de douleur plus élevé}

Tous les interprètes témoignent de cette grande familiarité avec le monde de la douleur.

«Bon, $t^{\prime}$ sais, en danse, on est quand même habitué de travailler avec la douleur là, on [ne] s'arrête pas juste pour dire «douleur», mais, bon...» (Lola). Lola est pourtant de celles qui pensent «que les danseurs élèvent un peu leur seuil de tolérance à la douleur...». Denyse, quant à elle, témoigne qu'elle va jusqu'à vomir tellement sa douleur d'accouchement est intense, sans même qu'elle ne la conscientise.

"Ça faisait vraiment très mal et puis à un moment, vraiment excrutiating mais, à un moment donné, j'ai vomi! et puis, j'ai dit à la sage-femme: «Mais, pourquoi est-ce que je viens de vomir?» Elle dit: «Mais parce que $t^{\prime}$ as mal, parce que $t^{\prime}$ as mal...» J'ai 
Douleur Chronique, usages du corps et souci et soi

dit: «Ah oui, j'ai si mal que ça?» [rit de bon cœur] (Denyse).

Plus que partout ailleurs, il apparaît clairement que la «douleur chronique» progresse selon une trajectoire spiralée dans le corps du danseur:

«Je pense que c'est la ténacité qui me tient et aussi, je crois que, parce que c'est pas tous les danseurs qui sont comme ça, mais, moi, j'ai une sensibilité, je résiste beaucoup à la douleur. Si j'ai mal, c'est parce que c'est sérieux parce que j'ai très, très mal, mais ça m'en prend beaucoup pour me plaindre. J'ai des petits bobos, tout ça; je la ressens la douleur, mais je [ne] vais pas me plaindre, je [ne] vais pas me dire: «Ah!!!» Si c'est intense, là je vais me plaindre; si c'est un choc.... » (Aurélie, interprète pigiste)

Il arrive même que les danseurs aient perdu la trace de l'événement déclencheur, tellement ils sont préoccupés d'en minimiser les effets sur leur carrière: «J'avais oublié, mais c'était, oui!!! Mais ça, je pense que c'était quelque chose qui m'a été fait par quelqu'un... Imagine, c'est bête, hein!» (Denyse). De toute évidence, ce que Denyse veut retenir de cette épreuve c'est qu'elle l'a traversée. Peu importe la cause ou l'origine, ce qui compte, c'est sa guérison! Avec tout le recul que lui donne sa victoire sur la douleur, elle souligne ainsi l'expertise et la grande connaissance intrinsèque du corps que développent les professionnels de la danse:

«Mais, y a des mouvements que je pouvais éviter parce que ça faisait mal et je place le poids différemment, en dansant, ou alors... j'arrête. Mais c'était... Donc j'arrêtais le mouvement... Si il y avait douleur, je $[n e]$ le faisais pas, donc, je $\left[n^{\prime}\right]$ avais pas mal...»

Denyse révèle ainsi une double limite, celle du seuil de la douleur et celle imposée au mouvement par l'apparition du moindre élancement. De 
cette façon, elle nous donne un début de réponse sur la place et le sens à accorder à la douleur jusqu'à sa guérison. Avec le temps et la pratique, Patricia a aussi développé sa propre théorie sur la valeur à accorder à la douleur et ce en quoi elle renseigne sur la limite à ne pas dépasser:

«La douleur nous dit: «Ah, qu'est-ce qui se passe? $\mathrm{Ah}$, je respire pas bien. Ah tiens, je déporte trop mon poids sur la gauche, Ah, tiens! Faut que je change mes souliers.» C'est ça qu'elle indique la douleur. Faut pas en faire son ennemie: c'est vraiment le signal. Quand on est danseur, depuis un bon bout de temps, vous l'avez peut-être entendu de la part des danseurs, ça devient ton repère, mais un moment donné, faut pas le nier non plus quand $c^{\prime}$ est aigu, c'est en crise, ton dos bloque, «c'est parce que là, il faut que t'arrêtes...»

Ainsi, même si les danseurs témoignent d'une endurance remarquable à la douleur, ils lui reconnaissent une valeur de signal que la limite est dépassée. Pour Aurélie, qui témoigne pourtant d'une endurance hors-pair, sans espoir de guérir un jour, la douleur joue un rôle de gardefou:

«Ben, elle sert à me protéger en quelque part, à pas faire des folies... Oui, oui, parce que moi, je suis assez kamikaze, donc elle sert, elle m'arrête en fait, sinon j'aurais pas de limites...»

Jeanne, pour sa part, a enduré sa douleur à la hanche pendant douze années. "J'attendais tant que je pouvais survivre, vivre avec ce problème-là. Même danser, c'est sûr que ce [ $\left.n^{\prime}\right]$ était pas facile. J'ai attendu, attendu, attendu...» Pour se faire, elle a créé sa propre compagnie pour choisir ses chorégraphes et pouvoir continuer de danser, selon ses capacités, sans que cela se sache, ni dans le milieu, ni dans son public. Elle a tout trafiqué pour que cela ne se voie pas:

«Ça $[n e]$ paraissait pas, sauf que, pour venir saluer, 
Douleur Chronique, usages du corps et souci et soi

je [ne] voulais pas venir saluer au milieu, je venais saluer par les côtés; je laissais les autres danseurs plus au centre pour [ne] pas que j'aie beaucoup de pas à faire, mais si je les faisais en courant, ça [ne] paraissait pas. J'ai tout trafiqué...»

Là aussi, Jeanne repousse les limites de la douleur le plus possible: «J'ai dansé finalement, mais à quel prix!» et «tout le temps en trouvant un chemin différent», en se faisant le moins mal possible et mais toujours en sachant «que c'est une hanche qu'il va falloir opérer...»

\section{La relation médecin-patient : un dialogue de sourds}

Le rejet de toute médication anti-douleur participe de la culture de l'endurance. La plupart des danseurs interrogés préfèrent en effet conserver la valeur alarmante de la douleur pour éviter d'aggraver le problème. Pour ces mêmes raisons, Sylvie, lors de la tournée en Allemagne, refuse de se laisser injecter quelque substance que ce soit. Elle a besoin de garder le contrôle sur ses sensations pour être capable de donner le show sans se blesser davantage: «I eventually went to see somebody in Germany, which was kind of an emergency thing, cause I had a show and he wanted to start shooting me with drugs, needles and I don't really want that...»10 Elle préfère de beaucoup s'exposer ainsi à la douleur plutôt que de l'occulter: «If I mask the pain, I understand doing that for a short period of time but, eventually, I want to figure out where the problem is so I can solve the problem instead of just covering up and forget about it» ${ }^{11}$ (Sylvie). Ansi, elle peut rester à l'écoute des moindres signes de détérioration et préserver son outil de travail.

De la même façon, Jeanne surprend beaucoup les médecins qui

10 «J'ai fini par aller voir quelqu'un en Allemagne, comme dans une espèce d'urgence parce que j'avais une représentation et il a commencé par vouloir m'injecter des drogues avec des aiguilles et ce n'était pas vraiment ça que ce que je désirais...»

11 «Si je cache la douleur, c'est pour une courte période mais ultimement, je veux savoir où se situe le problème afin de pouvoir le résoudre plutôt que de simplement l'enfouir et l'oublier.» 
mesurent l'intensité de la douleur à la quantité d'antalgiques consommés plutôt qu'au ressenti énoncé. Quand ils lui demandent, en consultation préopératoire: «Qu'est ce que vous avez pris comme médication?», et qu'elle répond: «Ben, un Tylenol, une fois de temps à autre», ils remettent en question la pertinence de l'intervention: «Vous êtes sure que vous avez besoin d'une opération à la hanche? Comment ça se fait que vous avez pu réussir à vivre pendant douze ans avec une hanche... Peut-être que votre hanche, elle $[n e]$ fait pas mal finalement, peut-être vous [ $\left.n^{\prime}\right]$ avez pas besoin d'être opérée.»

C'est bien mal connaître le milieu de la danse et son endurance à la douleur, que d'évaluer son intensité par la quantité de médicaments consommés. Car s'il est bien un trait commun à toutes et tous, c'est le rejet des médicaments anti-inflammatoires ou antalgiques qui modifient les sensations et posent le risque d'aggraver involontairement une blessure: «It's just killing the pain, I believe pain killer?» ${ }^{12}$ (Sylvie). La seule à faire exception et à en prendre encore régulièrement, Aurélie, est aussi la seule à endosser pleinement la catégorie douleur chronique et son irréversibilité supposée:

«Je [ne] prends pas de la cortisone tout le temps. Je $\left[n^{\prime}\right]$ en prends jamais sauf en cas de douleur intense ou d'extrême urgence. Fais que là c'est un peu ce qui se passe. Donc, suite à ces blessures, j'ai continué à danser, mais en ayant des traitements, régulièrement suite aux blessures et après ça les traitements de moins en moins jusqu'à temps que je [ $\left.n^{\prime}\right]$ ai plus besoin de traitement, voilà.»

Quelles sont les alternatives? Arrêter de danser? Souvent, c'est ce que conseillent les médecins: «Ben, écoutez si j'étais vous, j'arrêterai de danser...», dixit le médecin de Louis. À une exception près, celle du médecin de Renée, lié par un contrat avec la même compagnie de danse dont elle est salariée:

«Ouais, j’ai été voir mon médecin. Il semblerait

12 «Ça calme juste la douleur, un anti-douleur j'imagine.» 
Douleur Chronique, usages du corps et souci et soi

qu'ils aient décidé de toutes parts que mon état ne $\mathrm{s}^{\prime}$ améliorerait pas et puis que, soit je reviens dans des conditions limitées et douloureuses... Il était d'une froideur, mon médecin... Alors, il me laissait le choix, mon médecin: «Soit tu recommences et puis tu prends des médicaments pour [ne] pas avoir mal et puis tu peux rechuter quand tu veux rechuter là, mais je [ne] te marque aucune limitation dans ton dossier comme ça ton employeur [ne] peut pas te faire de problème» Et puis d'un autre côté, mon employeur me disait qu'il voulait un papier du médecin comme quoi j'étais $100 \%$ sans limitation. Donc, le médecin est d'accord de me faire un papier sans limitation comme ça l'autre il me prend, mais moi je fais quoi là-dedans? Parce que moi je le sais que je suis limitée, je le sais que j'ai mal. Qu'est-ce que je fais?» [Renée en arrêt de travail depuis plus de six mois.]

Ce médecin semble pousser à la reprise du travail sous médicaments, quitte à attester de la consolidation des lésions sans plus de preuve objective et sans tenir compte davantage des dires ni de la demande de Renée et de ses besoins. Le médecin semble pris alors dans la double contrainte de préserver son emploi à la danseuse et de satisfaire aux exigences de leur employeur commun, quitte à attester faussement de son état de santé. L'objectif visé semble être le retour au travail coûte que coûte et non plus l'acte thérapeutique, comme si la limite était imposée par l'employeur et non plus par l'éthique médicale.

La sagesse la plus élémentaire suggère en effet de s'abstenir de tout geste douloureux, respectant ainsi la fonction alarmante de la douleur. De la même façon, Sylvie relate que les médecins vont, soit lui conseiller d'arrêter de faire ce qui fait mal, soit de se reposer, soit encore d'arrêter de danser. Mais aucune de ces propositions ne répond à ses besoins: «[...]that's a difficult thing for a dancer to do or it is difficult for me to do because I have a contract, I have a show that I want to get to, and yes, if it is 
serious, of course, you need to stop.» ${ }^{13}$ Elle les soupçonne de lui conseiller d'arrêter par simple incapacité à poser un diagnostic, donc par incompétence. Elle renonce même à les consulter, car ils ne lui sont d'aucun secours, étant donné leur méconnaissance des spécificités du métier:

«But, until you get to the focus of what exactly the injury is and what the problem is, just stopping as a general practitioner often tells you to do, it doesn't help you, it doesn't get you better, it doesn't solve your problem or help you be focused in the good direction to solve the problem, the injury problem.» ${\text { (Sylvie, pigiste })^{14}}^{14}$

Elle préfère s'en remettre à un médecin du sport qui lui semble plus spécialisé et plus à même de circonscrire le problème, de poser un diagnostic et de proposer des solutions concrètes: «I know that a general practitioner does not have the answers that I'm going to need. One that's specialised in dance will definitely be able to send me in a better direction». ${ }^{15}$

Les médecins les plus appréciés dans le milieu ne suggèrent jamais d'arrêter de travailler en première intention, mais facilitent plutôt l'accès à des examens pour localiser le problème ou prescrivent des antalgiques ou des corticoïdes pour mieux continuer. Il apparaît clairement ainsi que la question du pouvoir dépasse largement le duel qui opposerait les savoirs experts aux savoirs profanes. Elle touche bien plutôt aux rouages d'un mécanisme qui rend efficaces ou non les injonctions et les méthodes

${ }_{13}$ «Mais c'est difficile pour un danseur ou alors c'est difficile pour moi parce que j'ai signé un contrat, j'ai un spectacle que je veux assurer et oui, si c'est grave, bien sûr, on doit arrêter...».

14 «Tant que tu ne sais pas exactement de quelle blessure il s'agit ni quel est le problème, te dire d'arrêter comme le fait souvent un médecin généraliste, ça ne $t^{\prime}$ aide pas, ça ne $t^{\prime}$ améliore pas, ça ne résout pas ton problème et ça ne $t^{\prime}$ aide pas à te concentrer dans la bonne direction pour résoudre le problème, le problème de la blessure».

15 «Je sais qu'un médecin généraliste n'est pas en mesure de répondre à mes besoins. Un spécialiste de la danse m'orientera certainement dans une meilleure direction». 
Douleur Chronique, usages du corps et souci et soi

d'assujettissement du comportement et des usages du corps.

«Car, nous sommes tous non seulement la cible d'un pouvoir, mais aussi le relais, ou le point d'où émane un certain pouvoir! Ce qu'il y a à découvrir en nous, ce n'est pas ce qui est aliéné, ou ce qui est inconscient, ce sont ces petites valves, ces petits relais, ces minuscules engrenages, ces microscopiques synapses par lesquelles le pouvoir passe et se trouve reconduit par lui-même» (Foucault dans Droit, 2004, p. 129).

De toute évidence, en faisant si peu de cas du vécu ou du ressenti et surtout des conséquences de ses prescriptions ou de ses injonctions sur la vie personnelle et professionnelle, le corps médical se voit largement décrédibilisé en matière de prise en charge ou de résolution des douleurs en lien avec les usages du corps dans le monde de la danse. D'ailleurs Denyse ne se gêne pas pour rejetter catégoriquement tout savoir médical: «Non; je suis pas du tout dans la médecine, moi, et j'y crois pas du tout!» Elle ne compte que sur elle-même. Au grand maximum sur l'aide d'un ostéopathe. Elle a ainsi développé une grande habileté dans les ajustements nécessaires à la continuation malgré les obstacles. Il est beaucoup question d'intelligence du corps, qui a appris de la douleur et qui, en bon soldat, continue de «performer», en évitant soigneusement la ou les zones à risque.

Forts de leur connaissance intrinsèque du corps, nombre d'interprètes continuent en effet malgré la douleur et construisent des déformations posturales d'évitement. Sylvie s'émerveille même de l'ingéniosité qu'elle attribue à un corps docile, quand elle se surprend en train de prendre appui avec la tête sur le placard de la cuisine pour faire la vaisselle sans réveiller la douleur $d u$ bas $d u$ dos, siège d'une hernie. Caroline convient quant à elle, sa part de responsabilité dans la gravité de la situation parce qu'elle a continué malgré la douleur.

«Ce [ $n^{\prime}$ ]est pas compliqué, mais du bas du coccyx jusqu'en haut... Tout avait [bougé]... Ben, il faut dire, j'avais eu le temps aussi, j'avais forcé, j'étais restée assise trois mois, donc [il] y avait eu le temps, 
Valérie Bloch

vraiment, qu' $\left[i l n^{\prime}\right] y$ ait plus rien qui corresponde.»

Ultimement, en cas de douleur insurmontable ou de blocage intempestif, les interprètes feront enfin appel à une aide extérieure non médicale. Dans le milieu de la danse, c'est unanime: une douleur en lien avec les usages du corps est du ressort des thérapies corporelles. Ce sont elles qui répondent le mieux aux besoins d'un corps blessé, malmené ou surmené (Perrault, 1988). Mais, après avoir rétabli la solution de continuité, l'alignement et le placement d'un corps désaxé ou surmené, encore faut-il consolider cet équilibre par de nouvelles attitudes, une nouvelle gestuelle et certaines limitations librement consenties:

«Donc, il m'a fait des petites manipulations, il m'a dit: «je vais te revoir à Paris, un moment donné. Je te conseille de reprendre très tranquille, [ne] fais aucun jeu de jambe en arrière, aucune extension avec des arabesques, aucun mouvement qui puisse agir dans le dos.» (Caroline).

«Il $\mathrm{m}$ 'a refait tout le taping pour tenir le bassin et puis, il m'a dit: «Non, tu [ne] fais pas tous ces mouvements là», tous les mouvements qui allaient par en arrière et puis, en fait, avec l'adrénaline et tout, j'ai fait le spectacle presque sans douleur. [rit]» (Lola).

\section{La fuite en avant : la « société du spetacle » 16}

La douleur fait donc partie intégrante du monde de la danse et donne rarement lieu à un arrêt de travail. Elle vient seulement s'ajouter aux autres contraintes d'horaires, de conditions de travail, de recherche de contrats ou de demandes de subvention. Elle est vécue comme une entrave

16 «En 1967, j'ai montré dans un livre, La Société du Spectacle, ce que le spectacle moderne était déjà essentiellement: le règne autocratique de l'économie marchande ayant accédé à un statut de souveraineté irresponsable, et l'ensemble des nouvelles techniques de gouvernement qui accompagnent ce règne» (Debord Guy, 1988). 
Douleur Chronique, usages du corps et souci et soi

comme une autre car, quoi qu'il arrive, il faut aller de l'avant:

«Pis la condition aussi était toujours liée, elle, à deux objets: un de pas faire sentir au chorégraphe qu'on est un poids mort dont il faut se débarrasser et puis, nous-même, pour nous conditionner à toujours avancer, parce que si on arrête là, on jette la serviette, $t^{\prime}$ sais...» (Patricia).

Sur scène, la loi est encore plus implacable. Lola s'y est retrouvée prisonnière et contrainte d'endurer la douleur pendant près d'une heure: «Il y avait les trois sur scène pendant une heure, de temps. Il n'y avait pas d'entrée, pas de sortie, je [ne] pouvais pas sortir. Donc, j'ai continué la danse jusqu'au bout...» Caroline témoigne quant à elle d'une sortie de scène à bout de bras, malgré une grave déchirure ligamentaire au genou et une douleur «atroce».

«Et là, je devais remonter rapidement et c'est ça, tout me picotait et je me suis dit:

«Comment je vais faire? Comment je vais faire?» Et là, je me suis dit: «Lève-toi!» Et là, j'ai fini le spectacle, mais vraiment, tout dans les bras, t'es vraiment... Là, t'es zoom out quoi... Tu vois le public et tu te dis... T'es comme: plus d'énergie, y a plus rien là...» (Caroline).

Même dans le meilleur des cas où des remplacements sont prévus, l'interprète doit toujours faire bonne figure lors de la représentation. «Même sur scène, c'est-à-dire, si jamais ça arrive, ben, tu sors de scène et puis, tu [ne] reviens plus sur scène» (Denyse). Denyse se bat pourtant avec ses danseurs pour qu'ils se ménagent pendant les répétitions:

«Non! Dorothée! T'as mal là aujourd'hui? Tu $\mathrm{m}^{\prime}$ as dit que $\mathrm{t}^{\prime}$ avais mal.»

«Non, Denyse! je veux le faire! Je sais exactement comment le contrôler, Denyse! Je [ne] vais pas faire tel mouvement, tel mouvement, tel mouvement...» 
Mais une fois sur scène, la même loi s'applique: «Gardez-le! Si vous tenez tant à le faire gardez-le! Mais, s'il vous plait, pas en répétition» (Denyse).

La culture de l'endurance rejoint alors la loi des apparences et de l'invisibilité. Même s'il va de soi qu'il vaut mieux s'arrêter en cas de blessure accidentelle, pour éviter d'aggraver la lésion, quoiqu'il arrive, priorité est néanmoins toujours donnée au spectacle: «The show must go on!» (Sylvie). Telle est la loi qui vient renforcer la culture d'endurance et qui contribue souvent à banaliser la douleur, l'inscrivant de façon chronique dans la mémoire corporelle. Dans bien des cas, c'est précisément l'endurance et la résistance à la douleur qui participent activement à sa chronicisation. Aurélie en est un parfait exemple puisqu'elle s'enorgueillit de sa résistance à la douleur et qu'elle choisit de vivre avec...

«Ben, comme mes douleurs: je me lève chaque matin et j'ai mal dans le dos, ben, je suis habituée, alors je vis avec, j'essaie de vivre en alliée avec... [rit] Donc, le fait de vivre comme ça, ça me permet de continuer à travailler.»

Arrêter, certaines l'ont fait quand elles en avaient le pouvoir, quitte à décevoir les attentes de leurs collègues et de leurs partenaires financiers. Lola, par exemple, s'est vue par la suite refuser des subventions et y voit un lien de cause à effet. Patricia, elle, n'ose même pas y penser et continue avec des Advil pendant un an, pour achever sa carrière en beauté. Dès qu'elle arrête la médication, la douleur revient. Elle est alors prise au piège:

«Je pleurais le matin, je me rappelle un moment donné c'est plus que ça faisait mal, c'est que ça [ne] s'en allait jamais... C'est que c'était récurrent, ça [ne] $\mathrm{s}^{\prime}$ en allait JAMAIS! Pis ce $\left[n^{\prime}\right]$ était pas non plus quelque chose dont on parlait beaucoup, les blessures en danse, aussi, pis la condition aussi était toujours liée, elle, à deux objets: un de [ne] pas faire sentir au chorégraphe qu'on est un poids mort, dont il faut se débarrasser et puis, nous-même, pour nous conditionner à toujours avancer, parce que si on arrête là, on jette la serviette, $t^{\prime}$ sais...» 
D'autres se font remplacer. Mais trop rares sont les compagnies qui prévoient un remplaçant en cas d'accident en cours de représentation et qui en ont les moyens. C'est alors très souvent sur ce non-respect de la valeur alarmante de la douleur que vient s'organiser le modèle «douleur chronique». Une douleur survient par exemple pendant ou après une représentation. Pour les besoins de la production, on passe au-dessus de la plainte, on la banalise, on la relativise. On invoquera bien sûr les rapports de la profession à la production, aux conséquences en termes de rentabilité de ce que l'on appelle désormais, les «arts du spectacle», l'industrie culturelle:

\begin{abstract}
«J'ai été blessée, il y a une année, au mois de juin 2009. On était en tournée et puis, j'ai commencé à avoir mal après un spectacle, je me suis fait soigner et j'ai refait un spectacle. Au bout de trois jours, on n'arrivait plus à récupérer, j'avais mal toujours à la hanche et là, on m'a un peu mis de la pression pour que je continue et ça s'est vraiment aggravé et j'ai dansé, j'ai dansé, là, en l'occurrence, en étant pratiquement incapable de marcher, je marchais avec des douleurs... Donc d'arriver à anesthésier ma douleur par un échauffement puis par l'adrénaline, pour pouvoir être sur scène, mais après, quand j'sortais d'scène, c'était terrible, j'étais handicapée ...» (Renée, salariée d'une grande compagnie de danse)
\end{abstract}

La relation employeur-salarié constitue certes le modèle explicatif le plus évident de l'endurance par soumission à l'autorité et à l'employeur, mais la motivation à poursuivre son activité coûte que coûte, repose aussi sur d'autres modèles. Cette loi implicite existe aussi chez les interprètes indépendantes qui, pourtant sans la pression d'un quelconque employeur, ont recours à des traitements de choc:

«Quand ça arrivait et que j'étais en choc donc, 
que je [ne] pouvais plus bouger, il y a un traitement, parce que le show doit continuer, hein, souvent ça arrivait, puis il fallait que je danse, il fallait que j'aille sur scène là, le soir même, donc, j'ai pris de la cortisone à très, très petites doses, à ce moment-là, donc... Je prenais pendant cinq jours de la cortisone et tout de suite la douleur, en une journée, même pas, la douleur baissait là, des 3 quarts, ce qui me permet de continuer, si j'ai un show à faire, de continuer...» (Aurélie).

«Continuer», s'oppose donc diamétralement à «arrêter» et dans le milieu de la danse, la fuite en avant est de rigueur. Denyse insiste beaucoup sur l'obstination de sa danseuse, Dorothée, qu'elle sait blessée, mais qui veut à tout prix continuer, bien que sa place ne soit pas du tout menacée. Des conditions de travail favorables ne suffisent donc pas à prévenir les blessures et à dissuader les interprètes elles-mêmes de prendre des risques. Bien souvent, l'injonction de continuer est implicite et repose sur plusieurs critères plus ou moins intriqués, mais néanmoins variables selon l'âge. Caroline, qui est aussi enseignante, révèle les lois liées à l'apprentissage et en lien avec la peur de perdre sa place, de faire mauvaise impression ou de témoigner d'un manque de maîtrise de son corps.

«Je devais avoir 16 ans, fais que là, j'ai continué à travailler, travailler, travailler... Et puis, aussi, tout un rapport à la technique ou l'habileté à forcer, parce qu'en fait, c'est ça aussi, dans l'apprentissage, surtout quand on apprend jeune, c'est aussi toujours lié à la compréhension de soi, donc du corps et de la volonté de bien faire et de répondre à des postures qu'on doit faire le mieux possible.» (Caroline).

Plusieurs d'entre elles témoignent en effet de la faible estime de soi de la débutante qui doit encore faire ses preuves, se construire un réseau, bâtir sa carrière et une image forte, endurante, infaillible, telle une machine. 
Douleur Chronique, usages du corps et souci et soi

\begin{abstract}
«Mais, moi, c'est ça, mais, peut-être que ça a eu un impact, mais je [n']ai jamais arrêté de danser, blessée, alors, peut-être que ce $\left[n^{\prime}\right]$ était pas une bonne idée, mais c'est toujours la peur de «on est toujours remplaçable», donc, si on se blesse et puis, on dit à l'employeur: «Oh! ben, je [ne] peux pas», ou, «je vais arrêter de danser», on se fait remplacer par quelqu'un d'autre, évidemment, ça coûte cher, les chorégraphes [ $\left.n^{\prime}\right]$ aiment pas ça; et là, ce $\left[n^{\prime}\right]$ est pas une belle image. Moi, j'ai toujours plus ou moins caché mes blessures ou été dans le minimum de déclaration que je m’étais blessée.» (Pénélope).
\end{abstract}

Pour les interprètes plus matures, il est plus souvent question d'impératifs financiers ou d'engagements moraux vis-à-vis des collègues et partenaires. «Il y avait un engagement aussi financier, une vente de billet, parce que c'était un partage de billetterie.» (Lola)

Peu importe alors, d'où vient la souffrance tant qu'elle n'empêche pas la performance. Le besoin de garder le contrôle sur les sensations physiques, de maintenir un fonctionnement et un rendement maximal, dans ces conditions, s'ajoute encore à la culture de l'endurance. «J'essayais de vendre mon spectacle» (Caroline). Quant à Louis, il témoigne de la grande précarité des petites et moyennes compagnies, quand pour sauver un salaire, le directeur est aussi le chorégraphe et le seul interprète:

«La douleur est devenue très grande, au point où je crois que, si j'avais été sage, j'aurai arrêté, mais [il] y avait toutes les impératifs financiers. J'avais cette responsabilité-là; c'est ma compagnie! Je [ $n^{\prime}$ ]avais pas les sous pour me faire remplacer alors $j$ 'ai décidé que j'allais mener le..., le bal...» (Louis).

Telle est la loi dans un monde désormais régi par des impératifs 
financiers et de production, dans une «société du spectacle». Face à la douleur, les issues sont certes plus ou moins heureuses, selon l'accessibilité aux soins, la flexibilité par rapport aux contrats et la nécessité de travailler selon les ressources financières et l'autonomie.

«[...]Avant d'être là-dedans y avait toute l'appréhension, la peur de, physiquement de... flancher, de se dire aussi que le spectacle qui est prévu demain aura-t-il lieu? Alors tu portes toute cette tension-là, je dirai, par rapport et tous les impacts financiers, parce que je suis responsable, je connais très bien le budget de la compagnie, je connais très bien la réalité.» (Louis, directeur de compagnie, interprète et chorégraphe).

Mais, en termes de vécu, toutes et tous sont égaux devant un phénomène qui échappe à l'entendement et au pouvoir médical puisqu'il renvoie essentiellement à une perversion d'un système à la solde de l'industrie du spectacle, qui met de l'avant la productivité et les contraintes budgétaires plutôt que l'essence de l'art.

L'endurance traverse donc tous les récits et s'impose comme ligne de subjectivation à tel point qu'elle en devient un trait culturel, au sens de pli ou de déformation professionnelle. Cette «culture» de l'endurance rend compte d'une dynamique professionnelle construite en grande partie sur un paradigme sociétal d'ordre économique. Il y est en effet question de «capital santé», $d^{\prime}$ «investissement» dans les soins corporels non remboursés et de «gestion» du stress. Le corps y occupe la place d'un corps-machine volontiers comparé à une voiture qui devrait battre des records de vitesse et ne jamais tomber en panne. Les récits des danseurs rendent compte d'un corps vécu comme inusable, infatiguable et corvéable à merci jusqu'à ce que la douleur les force à changer de regard et de comportement à son égard. La dualité corps-esprit fait alors place à une certaine complicité, voire un respect et une écoute des besoins du corps. Comme Jeanne qui parle désormais de son corps comme d'un outil de vie dont elle a appris à respecter le rythme. 
Douleur Chronique, usages du corps et souci et soi

\section{La guérison}

Enfin, près de la moitié des récits témoigne d'une guérison qui contredit la notion d'irréversibilité implicite contenue dans le qualificatif de chronique. Un maître-mot revient alors dans tous les récits et rejoint finalement l'injonction médicale d'arrêter. Car, une fois la blessure installée et conscientisée, la guérison passe par une immobilisation et bien souvent une reconfiguration des usages du corps. Or la décision d'arrêter revient le plus souvent à l'interprète qui est son propre employeur et tient trop souvent compte des conséquences d'un arrêt de travail sur sa carrière: «Mais un moment donné, faut pas le nier non plus quand c'est aigu, c'est en crise, ton dos bloque, c'est parce que, là, il faut que t'arrêtes...» (Patricia)

Cette attitude relève finalement d'un retour de savoir, de l'ordre d'un «savoir différentiel, incapable d'unanimité et qui ne doit sa force qu'au tranchant qu'il oppose à tous ceux qui l'entourent» (Foucault, 1997:9), et dont se prémunissent les danseurs et les danseuses. Car l'endurance y côtoie le plus souvent une grande sensibilité qui va de paire avec une grande connaissance intrinsèque du corps. Ainsi Denyse décrit une très grande tolérance à la douleur en même temps qu'une sensibilité très fine qui lui permet de transformer sa gestuelle dès que surgit la moindre sensation douloureuse. Son récit témoigne d'une combinaison gagnante entre une détermination très forte qui s'apparente à de l'endurance et une sensibilité de plus en plus raffinée qui semble être la clef d'une guérison qui passe par la transformation des usages du corps en adéquation avec les signaux douloureux. Elle s'oppose alors en tous points aux théories qui prévalent actuellement dans le monde de la recherche en santé et sécurité au travail et qui voudraient ignorer les sensations vécues comme douloureuses pour se concentrer uniquement sur l'aspect fonctionnel et le retour au travail. La douleur chronique y est considérée comme un problème de tolérance à la douleur et assimilée à un trouble du comportement, qui serait à rééduquer. L'objectif des thérapies issues de ce modèle n'est d'ailleurs plus la disparition de la douleur, mais bien plutôt le retour au travail sans égard à la douleur perçue (Coutu M.-F. et al., 2010). La guérison ne consiste plus alors en la disparition du signal douloureux, mais en un retour à la productivité. Là encore les définitions et les énoncés se transforment au gré des besoins du monde du travail témoignant de la confusion qui règne dans le champ thérapeutique. Plus que jamais, il est 
clair que la danse et la médecine exercent souvent le pouvoir selon une même ligne de force, celle de l'endurance, afin de répondre aux exigences de productivité et de fonctionnalité des sujets, qui n'ont d'égales que celles de nos sociétés néolibérales.

Ainsi l'endurance présente dans chacun des récits est le reflet de relations de pouvoir multiples. De même que le biopouvoir ne peut s'exercer qu'avec la participation implicite du sujet, ce dernier exerce aussi des relations de pouvoir sur son environnement affectif ou professionnel et sur lui-même. En effet, il n'y a pas que les médecins, spécialistes de la douleur ou de la danse, ni les physiothérapeuthes qui intiment à celles et ceux qui souffrent de continuer à fonctionner comme si de rien n'était... Ce sont aussi les chorégraphes, les directeurs artistiques et les danseurs et danseuses eux-mêmes, sous l'influence de paradigmes productivistes et d'une représentation duelle du corps et de la modernité. Les différents récits révèlent davantage un souci excessif de "performer», voire de transgresser ses limites corporelles, qu'un respect des besoins élémentaires du corps-sujet de se reposer, de se ressourcer à intervalles réguliers, de prendre son temps. Les lois du silence et de l'invisibilité, combinées aux enjeux financiers et de carrière viennent renforcer une qualité d'endurance qui honore les interprètes, mais qui, simultanément, crée les conditions de la chronicité en même temps qu'elle s'oppose à la guérison.

La généalogie des évènements qui mènent vers la guérison incite donc à mettre davantage la zone douloureuse au repos et ce, quitte à suspendre toute activité douloureuse, voire professionnelle, au lieu de lutter pour repousser les limites corporelles et accroître l'endurance ad vitam aeternam.

Le secret de la guérison ne réside donc pas tant dans une façon de faire unique et universelle, à inscrire dans les guidelines. Six études de cas de guérison permettent de penser qu'elle est plutôt le fruit d'un long processus de transformation tout en douceur qui signe la résolution d'une situation de crise, quand les danseuses retrouvent du pouvoir décisionnel sur les usages de leur corps. Dans chacun de ces cas, le corps et, à travers lui, le sujet, témoigne d'un changement d'attitude à la fois physique et mentale. Pour initier le changement nécessaire à la guérison, il faut prendre un certain recul, luttant ainsi contre ce courant qui voudrait que l'on aille toujours de l'avant.

L'arrêt des usages douloureux n'est donc pas définitif, mais 
Douleur Chronique, usages du corps et souci et soi

nécessaire pour prendre conscience des changements nécessaires d'orientation, soit professionnelle, soit gestuelle. Ainsi Lola a fini par se dire: «Ben, si je veux danser c'est comme, ben, là, c'est le temps d'une grande transformation.» Et après trois années d'interruption, elle a changé sa façon d'initier le geste, sous l'influence du butô:

«Bon, une chose qu'il dit souvent c'est comme le mouvement doit vraiment venir du coeur, donc, peut-être, des fois, c'est mieux de commencer par petit, et que ça s'exprime vraiment par le coeur et puis, après, ça peut s'étendre et dans ce sens-là, j'ai travaillé beaucoup dans ce sens-là...»

Pour Patricia, la douleur chronique vient clairement indiquer le besoin de faire le point, de mettre fin à sa carrière et de prendre du temps pour elle. Caroline, quant à elle, part en voyage sans même considérer les indemnités auxquelles elle aurait pu prétendre, afin de faire le point sur sa carrière et sur sa vie. C'est à ce prix qu'elle réussit à reconnecter son corps à son état d'esprit, à retrouver son élan vital et sa force créatrice. C'est comme si la blessure l'avait ramené à l'essentiel. Sylvie a préféré reprendre des études pour changer complètement de milieu avant de revenir à la danse. Cette coupure lui a permis de comprendre combien elle était sensible à l'environnement affectif et à l'ambiance de travail. Même si elle est la seule à ne pas avoir créé sa propre compagnie, elle a décidé de rester pigiste pour ne pas avoir à subir trop longtemps des conditions qui la mettraient à risque de se blesser si cela devait encore lui arriver. Seule Jeanne a continué, mais en créant sa propre compagnie et en gardant le contrôle sur la chorégraphie. Ainsi sa danse s'est enrichie de toutes les nuances que cette épreuve et ses limitations lui ont révélé. Tout son cheminement a des allures d'initiation et désormais, elle se sent de mieux en mieux équipée face aux épreuves de la vie: «Après ces douze ans-là, où j'ai travaillé avec un problème, j'ai gagné cette patience, de [ne] pas m'affoler avec quelque chose qui [ne] va pas.» Son regard sur le corps a changé et participe d'une nouvelle attitude indissociable de la guérison. D'outil de travail qu'il était, son corps est devenu un «outil pour goûter la vie». Jeanne a appris à le suivre plutôt qu'à le malmener. Cette blessure, en lui révélant sa vulnérabilité, l'a sans doute éveillée à d'autres dimensions 
de la danse. Dès lors, elle a cessé de se battre avec son corps et de lui imposer sa volonté. Sa passion pour la danse l'a obligée à explorer d'autres pistes, des chemins de traverse, et à devenir maître de sa danse. La guérison apparaît ainsi avant tout comme le fruit d'une attention redoublée aux besoins du corps et à l'acquisition d'une connaissance de plus en plus fine de ses limites: «Je laisse le temps au corps, il a besoin d'un temps de récupération et puis, mon corps me donne beaucoup, il récupère vite, mais il faut pas que je veuille aller plus vite que déjà le court temps qu'il me demande, faut pas que je lui en enlève dessus...» (Jeanne).

La douleur résultant de la répétition d'un même geste incite donc à modifier les usages du corps de toutes celles qui ont guéri: «Ben, aussi, parce que au bout d'un moment aussi, on apprend à plus faire, ce qui fait mal, quand même! je veux dire faut pas être idiot.»(Caroline). Ainsi la suspension précoce des usages du corps en lien avec la douleur initiale et qui lui conserve une valeur de symptôme ou de limite à ne pas dépasser, paraît être une alternative que Lola explore dans sa phase de rééducation.

«Non, quand j'étais, comme en réhabilitation, là, j'essayais vraiment d'éviter la douleur. Je $\left[n^{\prime}\right.$ ]essayais pas de faire des mouvements pour tester si c'était pour faire une douleur ou non, parce que je [ne] voulais pas, comme reproduire la même chose et puis, retomber en inflammation et puis, en spasme, fais que... C'est ça, quand j'étais dans la piscine et tout, des fois, si j'essayais quelque chose et puis, j'étais à l'écoute, si je sentais comme une petite pointe là, comme de la douleur, j'arrêtais et puis, je [ne] faisais pas ce mouvement là et puis, je $[n]$ 'essayais pas de le faire.»

À travers les récits, il apparaît toutefois qu'une activité modérée est permise, à condition de ne pas reproduire le geste ou l'usage qui a mené à la blessure initiale:

«C'est que, aussi, moi, je pense que si on fait toute sa vie des arabesques et puis un moment donné on se rend compte que, non seulement, on a pas de 
Douleur Chronique, usages du corps et souci et soi

plaisir à faire ce mouvement-là, mais qu'il est pas nécessaire dans la danse...»(Caroline)

Celles qui s'en sortent ont donc réussi à réconcilier leurs aspirations avec leurs possibilités. Elles ont fini par accepter et même respecter les besoins de leur corps quitte à se réorienter professionnellement après avoir mis fin en beauté à leur carrière. Mais, s'il est un fait particulièrement remarquable, c'est qu'aucune des guérisons ne s'est opérée grâce à des remèdes pharmaceutiques et surtout pas des anti-douleur. Car la guérison s'appuie aussi sur des savoirs universels qui valorisent le ressenti et en particulier la douleur qui conserve ainsi toute son utilité. Sa valeur de signal reste essentielle, en termes de préservation et de limitation des dégâts, quelle que soit sa durée. «Ben, aussi, parce que au bout d'un moment aussi, on apprend à [ne] plus faire, ce qui fait mal, quand même! Je veux dire: «Faut pas être idiot!»» (Caroline). Celles qui ont guéri, malgré une endurance hors du commun, dont nous avons vu qu'elle est plutôt un obstacle à la guérison, démontrent surtout une connaissance subtile et un souci du corps proche d'un souci de soi (Foucault, 2001).

Ainsi Denyse insiste sur la répartition adéquate du poids du corps pour protéger la zone blessée. Lola et Jeanne décrivent leur nouvelle façon de s'élancer et d'initier le mouvement. Sylvie apprend à se protéger d'environnements professionnels à risque de provoquer des émotions dévastatrices sur le travail corporel. Toutes témoignent d'une relation au corps plus harmonieuse et d'une transformation de ses usages. Pour Jeanne, il passe d'outil de travail à outil de vie. Toutes celles qui ont guéri se sont appuyées sur leur connaissance intrinsèque du corps et leur ressenti et ont banni les «anti-douleur» pour guider leur démarche thérapeutique et leur réorientation de carrière, passant d'interprète et chorégraphe à interprète solo ou seulement chorégraphe ou encore en changeant de carrière. Ainsi, c'est aussi par la force de l'art, au sens où l'entend Henri Barras comme étant «du domaine de la sensation, des choses ressenties», que les interprètes ont pu accéder à la guérison (Barras, 2007).

En abordant la problématique d'une douleur chronique en lien avec les usages du corps et de ses mécanismes au creux du sujet en termes de vécu et de ressenti, j'ai souhaité redonner corps à ce que la médecine disqualifie le plus souvent en le taxant de «subjectif». L'injonction de baser sa pratique sur des évidences convie en effet la plupart des médecins à 
soumettre à caution tout ce qui est du domaine de l'invisible ou de l'impalpable et à fortiori une douleur incompréhensible qui a la réputation de résister aux traitements habituels. Pourtant, c'est en suivant les trajectoires personnelles, interpersonnelles et contextuelles qui mènent à une douleur «pas comme les autres» que j'ai pu leur retrouver un sens et constater que la guérison, quand elle s'opère ne le doit que rarement à la médecine et certainement pas aux antalgiques. Elle procède plutôt d'une grande transformation (Lola) et d'une insurrection des savoirs, d'un changement de posture physique et mentale. Celles qui ont guéri se sont situées d'emblée à distance d'un monde médical qu'elles ne semblent pas tenir en grande estime. De fait, en déplaçant le champ thérapeutique, de la médecine vers les thérapies corporelles et en renonçant catégoriquement aux «anti-douleur», les danseuses guéries tracent les lignes d'un autre dispositif, celui de la guérison, selon lequel circulent des savoirs d'un autre ordre. Elles semblent alors munies du pouvoir critique de celles et ceux qui possèdent des savoirs enfouis, des savoirs universels, mais «assujettis», qui viennent confronter le pouvoir en place, celui de la science:

«Par «savoirs assujettis», j'entends également toute une série de savoirs qui se trouvent disqualifiés comme savoirs non conceptuels, comme savoirs insuffisamment élaborés: savoirs naïs, savoirs hiérarchiquement inférieurs, savoirs en dessous du niveau de la connaissance ou de la scientificité requises. Et c'est par la réapparition de ces savoirs d'en dessous, de ces savoirs non qualifiés, de ces savoirs disqualifiés même, c'est par la réapparition de ces savoirs: celui du psychiatrisé, celui du malade, celui de l'infirmier, celui du médecin, mais parallèle et marginal par rapport au savoir médical, le savoir du délinquant, etc.- ce savoir que j'appellerais, si vous le voulez, le «savoir des gens» (et qui n'est pas du tout un savoir commun, un bon sens, mais au contraire, un savoir particulier, un savoir local, régional, un savoir différentiel, incapable d'unanimité et qui ne doit sa force qu'au tranchant qu'il oppose à tous ceux qui l'entourent) - 
Douleur Chronique, usages du corps et souci et soi

c'est par la réapparition de ces savoirs locaux des gens, de ces savoirs disqualifiés que s'est faite la critique» (Foucault, 1997: 9).

\section{Le Sens}

Une des questions centrales reste alors celle de la place à accorder à la douleur en lien avec les usages du corps. Mais, auparavant, il importe de s'attarder sur ces douleurs qui disparaissent secondairement, comme par enchantement et tendent à faire croire qu'elles étaient imaginaires parce qu'elles nourrissent les a priori qui en font d'emblée une nouvelle forme d'hystérie et renforcent le socle d'une catégorisation arbitraire. Telle que la décrivent Denyse et Patricia, la résolution de ces douleurs ressemble davantage à une «épiphanie», une prise de conscience tellement forte et soudaine qu'elle laisse croire à un effet de la volonté ou à un «miracle». Il s'agit là de sensations vécues comme douloureuses, qui font suite à de réelles blessures et qui sont à comprendre comme des traces mémorielles ou simplement comme une surinterprétation de sensations reliées à une zone vécue comme «à problème» pendant trop longtemps. Ce phénomène persiste alors que la fonction de signal n'a plus lieu d'être. Il s'agirait d'une sorte de boucle réflexe protectrice d'une zone encore vécue comme vulnérable par la force de l'habitude. Ces douleurs ne correspondent plus à aucun substrat anatomique et disparaissent au moment opportun, une fois le travail de réparation et de reconstruction finalisé. Elles peuvent à raison être qualifiées de douleurs sans objet, secondaires aux douleurs originelles qui traduisaient de réels problèmes d'ordre musculosquelettiques le plus souvent. Il serait donc tout à fait regrettable d'en déduire pour autant que toutes les douleurs qui durent depuis plus de trois mois ne sont que des douleurs «fantômes» et ne seraient que des «vues de l'esprit».

Pour Louis, la douleur est bien réelle et fait irruption dans la danse, mais il a appris à la gérer et même à l'intégrer dans sa performance. La douleur y joue néanmoins le rôle d'aiguillon qui le ramène à la terre et lui rappelle sa condition:

«[...]jusqu'au moment où j'allais faire le spectacle qui était toujours un moment où vraiment je partais 
là, j'oubliais les problèmes de genou, ça [ne] faisait pas partie du scénario là, sauf des fois, quand ça faisait très, très mal, à des mouvements précis là: Ah! [fais la grimace] Mais il fallait que je me ressaisisse. C'est arrivé plusieurs fois en spectacle que j'ai eu des douleurs, quand même aiguës, alors comment tu vis avec ça? Ben, tu essaies, O.K., tu essaies de respirer puis de ralentir le rythme, puis après, la douleur est passée, tu reprends...»

Les ouvrages spécialisés soulignent combien le stoïcisme, et l'art de souffrir en silence, sont considérés comme des traits de caractère très positifs en contexte occidental (Rey, 1993). Celles et ceux qui ne sont pas encore guéris témoignent d'ailleurs d'une lutte constante avec une douleur qu'ils ne veulent pas écouter. Leurs récits donnent l'impression d'un duel corps-esprit entre le désir de danser, l'aspiration à cet état second dans lequel leur performance les plonge et le plaisir d'être sur scène.

«C'est une lutte constante entre mon mental et mon corps, j'ai l'impression. Alors, c'est difficile parce que c'est sûr que pour avancer j'suis obligée de pousser un petit peu plus mon corps, mais lui il rouspète après. [Alors] j'suis obligée d'attendre...C'est un dialogue que je dois faire qui est très... Comment dire... Je dois vraiment être à l'écoute des deux là... Parce que je [ne] sais pas si mon corps va être d'accord de collaborer jusqu'au bout...» (Renée).

«Alors, c'était tout ça qui était très, très dur à accepter, enfin mais $c^{\prime}$ est juste parce que j'ai dû rester assis avec les sacs de glace pis les antidouleur, les anti-inflammatoires que j'ai compris que... Et c'est comme j'ai dit ça m'a vraiment isolé là, c'était vraiment je dirai une crise, une crise de croissance, c'était vraiment ça, je vivais au- delà de la douleur. La douleur a provoqué cette crise de...je dirai de 
Douleur Chronique, usages du corps et souci et soi

croissance, de passage de dire « ben voilà mon pit, $y$ va falloir que tu acceptes que dans la vie on vieillit, et que ça a des conséquences, le métier que je fais a des conséquences et un jour il faut payer ». Et la douleur, comme je t'ai dit, c'est dans le moment où tu danses vraiment tu en fais, moi, j'en faisais abstraction...C'était vraiment ça et puis ça je considère que ça c'est comme central. La douleur tu peux la gérer par les médicaments, tu peux la gérer par ta force mentale, mais elle $t^{\prime}$ attend toujours à la sortie du théâtre, là c'est le cas de le dire...» (Louis).

La douleur chronique s'inscrit donc dans une dynamique conflictuelle de l'ordre de la résistance passive. On pense à l'arme du faible, «the weapon of the weak», celle d'un corps qui s'opposerait à l'esprit en contexte de représentation duelle de la relation corps-esprit (Scott, 1985). La douleur chronique vient alors s'immiscer dans l'exercice de l'art du danseur professionnel et transforme, ce qui se voulait un travail, en combat. Le récit de Louis illustre bien le conflit à l'oeuvre et la résistance au changement qu'objective cette douleur insistante et ce, malgré toutes les dérobades du sujet, qui maintient son activité physique coûte que coûte au mépris de tous les «cris du corps».

«Fais que, c'était finalement un combat contre moimême, c'était, je dirai, le combat contre la douleur était un combat contre moi-même. À accepter que j'ai mal. À accepter que je devais arrêter. Je n'avais vraiment pas envie. Ça a été un cheminement d'un an et demi. J'ai continué à danser même avec la douleur, avant que je dise: «Faut que j'arrête!»» (Louis).

\section{Une crise existentielle et sociale}

La douleur chronique plus que tout événement en lien avec les usages $\mathrm{du}$ corps pousse le sujet dans ses derniers retranchements et témoigne décidément d'une véritable crise du sujet. 
«C'est vraiment, c'est le mental qui... Et je dirai, pas juste le mental... Mais, c'est sa capacité à gérer cette douleur-là et à l'accepter parce que sinon, y a un moment donné, je me suis dit: «C'est assez! J'en ai assez d'avoir mal! J'adore être devant le public, c'est comme «J'me sens chez moi!» Mais en même temps il faut, à un moment donné, se retirer parce qu'il y a d'autres enjeux»(Louis).

Car Louis en convient, la décision d'arrêter et d'initier un changement vient avant tout de soi, plutôt que du monde médical. Une étude approfondie des tenants et des aboutissants d'une douleur, perçue tout d'abord comme un signe de rébellion, révèle combien elle vient confronter chacun et chacune dans ses croyances et dans ses valeurs les plus fondamentales. Les limites imposées aux usages du corps et à l'essence de l'art créent les conditions d'une crise existentielle profonde. Sa résolution et la guérison ne peuvent alors advenir qu'au prix d'une bascule du même ordre et donc d'un renversement des forces en présence. C'est d'ailleurs bien souvent l'arrêt forcé qui initie le processus de guérison. Qu'il soit choisi volontairement ou imposé par les circonstances, l'arrêt procède $\mathrm{du}$ renversement des forces en présence au sens où tous les usages professionnels du corps sont suspendus. Le sujet est alors livré à lui-même et aux effets de la douleur en dehors de tout contexte professionnel. C'est alors le temps de la confrontation avec ses priorités et avec sa dépendance vis-à-vis d'un corps qui ne répond plus aux commandes habituelles. La guérison exige de reconsidérer ses motivations, de questionner ses centres d'intérêt et plus subtilement ses intentions et le sens à donner à ses faits et gestes. C'est le temps d'une prise de conscience et «d'une grande transformation» [Lola]. Le processus de guérison passe inéluctablement par un arrêt des activités nécessaire et suffisant, pour permettre de dégager les forces en présence et de les réorganiser. Le sujet y exerce alors son pouvoir en transformant un arrêt de travail, souvent vécu comme obligé, en opportunité de repenser les usages de son corps et sa gestuelle. Parce que la douleur chronique correspond le plus souvent à une crise profonde du sujet, sa guérison nécessite la transformation du point de vue sur le corps qui devient désormais un égal plutôt qu'un subalterne, que ce soit au 
Douleur Chronique, usages du corps et souci et soi

service de l'art ou de l'esprit. La douleur exige de repenser les usages de son corps en même temps que ses conditions de travail et la qualité de ses relations professionnelle et thérapeutique, parce que «même au sein de la définition de la maladie comme une pathologie reliée à un organe, la maladie n'est pas une entité à part entière mais un concept relationnel.» (Eisenberg, 1977: 18).

Si une approche généalogique de la douleur permet d'en révéler les racines souvent profondes et anciennes, une écoute attentive de la plainte rend compte à son tour de la multiplicité des enjeux et de la complexité des dynamiques de pouvoir sous-jacentes. Ainsi une prise en charge précoce d'une douleur en lien avec les usages du corps devrait suffire à éliminer les douleurs résiduelles sans objet et toute catégorisation chronique. Bien sûr, cela impliquerait d'inscrire une entrevue d'une durée minimale d'une heure et demie guidée par une écoute sensible et neutre dans les guidelines, ce qui serait en soi une révolution... En observant les usages d'un corps perçu avant tout comme un outil de travail, j'ai pu déceler les «plis» d'ordre socio-culturel tels que l'endurance, mais il est une loi d'un autre ordre, celle de la productivité dans laquelle s'inscrivent ces douleurs «pas comme les autres». Ainsi en contexte post-industriel, les contraintes financières dessinent une ligne de force qui traverse autant la dynamique personnelle du sujet que le milieu professionnel et la société toute entière. Cette loi contribue largement à relativiser les sensations douloureuses et à encourager une fuite en avant qui ne fait qu'aggraver une situation qui exige plutôt de s'arrêter pour faire un état des lieux et un réajustement des usages du corps. Malheureusement, le monde médical et le monde du travail semblent avoir adopté à cet égard les mêmes exigences de rentabilité au détriment de celui ou celle qui souffre. Ainsi, donc, le comportement de déni inscrit, autant dans le milieu de travail que dans la société environnante et dans le monde médical, renforce le risque de chronicité. Qui plus est, les usages d'un corps perçu davantage comme un objet perfectible et malléable, soumis à la force de la bonne volonté aussi bien par le sujet dansant que par l'employeur ou le thérapeute l'exposent davantage aux blessures d'usure et aux accidents.

Ramené au plan sociétal, le phénomène douleur chronique semble désigner aussi les limites d'un système basé sur une productivité à tout crin de plus en plus friand de nouvelles technologies et calqué sur celui du rouleau compresseur et de l'exclusion. La douleur chronique signale 
définitivement un problème en lien avec les usages du corps qui, avec le temps, mène à l'immobilisation. Elle fait donc office de grain de sable dans la machine économique comme dans la machine physique. Elle vient empêcher de faire comme si de rien n'était et même si l'institution médicale tend à la réduire au silence, en prescrivant des antalgiques de plus en plus apparentés à des anesthésiques, le problème sous-jacent continue son œuvre d'obstruction et vient alourdir la facture des indemnités de salaire et des traitements.

\section{La douleur chronique comme operateur de changement}

La douleur chronique se construit soit sur l'accident, ici et maintenant, la chute, l'effet d'un décentrement, d'un échappement, soit sur la répétition d'un même geste, d'un même usage du corps, bref, elle surprend: «I was in rehearsal and I slid, it was hot and I was holding on to somebody so...» ${ }^{17}$ Pour Caroline, «c'était surdimensionné, cette blessure par rapport au mouvement que j'avais à faire.» Pour elle, «[...]le rapport aux blessures chroniques, en tout cas, c'est lié à l'état d'esprit, c'est sûr, à la disponibilité présente...» C'est une question de présence à soi et d'écoute des besoins du corps, mais comme elle le dit si bien: «On ne peut pas toujours être en contrôle.» Mais, une fois l'accident arrivé, la douleur donne souvent lieu à des stratégies de l'ordre du déni ou de l'évitement qui, de fait, en prolongent l'effet.

Ainsi, en examinant de plus près les lignes de subjectivation, selon lesquelles le pouvoir s'insinue dans le corps du sujet, j'ai pu observer comment chacun et chacune exerce à son tour le pouvoir d'abord selon un modèle dominant-dominé puis, selon celui de la complémentarité, de l'équité et $\mathrm{du}$ respect des rythmes réciproques, du dialogue et de la réconciliation qui mènent à la guérison. À ce moment crucial, le centre de gravité du pouvoir se situe au cœur du sujet, comme le geste dont parle Jeanne et qui permet le retour à l'équilibre et au juste milieu: «J'allais pas pour faire trop, trop, trop, tout le temps, j'allais au coeur du mouvement, $c^{\prime}$ est toujours le coeur et puis, le chemin» [Jeanne].

Mais si le pouvoir s'applique au corps afin de rendre le sujet docile,

17 «J'étais en répétition et j'ai glissé, il faisait chaud et je me tenais à quelqu'un alors...» 
Douleur Chronique, usages du corps et souci et soi

le corps, et à travers lui le sujet, détient lui aussi les rênes du pouvoir et le prend, soit pour mieux se soumettre à l'autorité, soit pour s'inscrire dans la chronicité, ou enfin pour trouver le chemin de la guérison: «Nous avons tous du pouvoir dans le corps» (Foucault, 1997: 27). Le corps humain est le lieu-charnière à travers lequel s'exercent les relations de pouvoir et de savoir visant à l'assujettir. Mais le corps est aussi, de fait, celui d'où peuvent s'initier les révolutions et les renversements de régimes. Le corps est à la fois le lieu de la résistance et le lieu du désir, le lieu par où ça passe et où ça se passe.

La douleur devenue chronique, par la répétition d'un même signal, semble envoyer un message clair, un appel insistant au changement, par n'importe quels moyens. Elle agit comme révélateur du seuil de tolérance à la douleur et questionne la relation entre le corps et l'être. Le récit de Jeanne en apporte le témoignage le plus extrême puisqu'elle a dansé pendant douze ans avec une douleur à la hanche sans que personne ne s'en aperçoive. Pourtant, elle a aussi développé un modèle d'endurance maximale en s'entraînant à travailler dans toutes les conditions: "Je sais comment travailler malade, je le sais, je me suis pointée au studio malade, j'ai trouvé une manière... Fais que, comme ça, tu renforces ton corps et puis t'apprends ...» Mais en même temps, elle s'est réapproprié les dynamiques de pouvoir qui conditionnent l'usage de son corps en quittant la grosse compagnie pour pouvoir choisir ses chorégraphes, elle a modifié sa gestuelle et sa relation au corps:

«Je laisse le temps au corps, il a besoin d'un temps de récupération et puis, mon corps me donne beaucoup, il récupère vite, mais il [ne] faut pas que je veuille aller plus vite que déjà le court temps qu'il me demande, [il ne]faut pas que je lui en enlève dessus...» (Jeanne).

Comme elle, toutes celles qui s'en sortent ont réussi à réconcilier leurs aspirations avec leurs possibilités. Elles ont fini par accepter et même respecter les besoins de leur corps. Mais Lola est la seule à énoncer clairement combien la douleur vient questionner profondément sa gestuelle et sa façon de fonctionner: «[...] et puis, ça m'a amené à me dire: «Ben, si je veux danser c'est comme, ben, là, c'est le temps d'une grande 
Valérie Bloch

transformation.»» Elle décrit parfaitement le changement d'usage du corps qui accompagne le changement de gestuelle et qui signe la guérison:

«C'est après cette création-là, en 2003, que j'ai recommencé à danser en solo, tranquillement, mais, vraiment, en adaptant les mouvements pour mon corps, sachant que je [ne] pouvais pas faire ci, je [ne] pouvais pas faire ça, mais, tranquillement, ça se solidifiait de plus en plus. Et puis, un moment donné, je pense que c'est en 2004, j'ai présenté la 1ère pièce avec [nom d'un danseur de butô japonais] et puis, vraiment, je peux dire que là, vraiment, je dansais et je [ne] pensais plus à ma hanche et ça, ben, c'est bon signe. C'est comme, je [ne] me préoccupais plus de: «est ce que je vais avoir mal?», parce que je savais que, ce que je faisais, les mouvements que je faisais, pourtant je bougeais quand même assez vite, mais, j'avais changé ma gestuelle et puis, ma façon de me déplacer dans l'espace et puis, bon, je veux dire, je [ $n^{\prime}$ ]avais pas l'air handicapée là, c'était juste comme une autre façon de bouger...» (Lola).

Caroline quant à elle a aussi créé sa propre compagnie et s'autorise ainsi à renoncer aux arabesques:

«C'est que, aussi, moi, je pense que si on fait toute sa vie des arabesques et puis un moment donné on se rend compte que, non seulement, on n'a pas de plaisir à faire ce mouvement-là, mais qu'il [ $\left.n^{\prime}\right]$ est pas nécessaire dans la danse...» (Caroline).

Denyse est passée d'interprète à directrice de sa propre compagnie et met un point d'honneur à partager sa grande connaissance des limites du corps et sa maîtrise du poids du corps à ses interprètes.

Sylvie a porté davantage son attention sur l'environnement affectif auquel son corps est très sensible. Même si elle est la seule à ne pas avoir 
Douleur Chronique, usages du corps et souci et soi

créé sa propre compagnie, elle est devenue pigiste pour mieux se protéger d'éventuelles ambiances qui la mettraient à risque de se blesser.

Chaque cas de guérison comporte donc son lot de changements, facteurs de résolution: changement d'activité, changement de statut, changement d'état d'esprit, changement des usages du corps, changement de carrière... La guérison repose donc toujours sur un changement de paradigme et de posture face à un corps qui révèle ses limites. La guérison se construit sur un rapport de pouvoir entre corps et esprit dont Renée est bien consciente même si sa propre démarche n'a pas encore abouti:

«Je me rends compte qu'il m'est encore très difficile d'écouter mon corps, très, très difficile ... Quand il me donne des petits signaux, j'ai du mal parce que je lui dis ... Je [ne] l'écoute pas, j'ai juste le goût de continuer ma vie tel que j'ai l'habitude de la faire et pis de $[n e]$ pas comprendre que mon corps vieillit, fatigue, devient moins résilient et qu'il a d'autres demandes...» (Renée).

Elle témoigne d'ailleurs d'une certaine ambivalence et d'un attachement à la douleur après un certain temps. Sa douleur devient alors presque existentielle comme chez Antonin Artaud dont Jacob Rogozinski fait remarquer que ses écrits recouvrent des symptômes reconnus désormais comme ceux de la fibromyalgie (Rogozinski, 2011):

«[...]de comprendre comment $c^{\prime}$ est devenu une part entière de moi et que continuellement quand je danse je vais aller recontacter la douleur pour vérifier... Pour vérifier quoi? C'est ridicule, mais...Elle a été tellement présente qu'elle fait partie de moi, que c'est un travail autant de mémoire du corps ... que de la tête et me détacher d'elle....si je veux le faire mon miracle il faut que je la laisse partir la douleur.»(Renée)

La douleur ramène le sujet à l'essentiel, pose la question identitaire et incite au changement comme en témoigne cet extrait de dialogue entre 
Valérie Bloch

Renée et sa douleur, issu d'une séance de sophrologie:

«Renée: Mais quand tu es arrivée, qu'est-ce qui se passait autour de moi?

La douleur: Ça bougeait, mais tu ne regardais pas. Ça ne bougeait plus en toi Renée. Autour de toi ça bougeait, mais tu étais figée.

Renée: J'étais figée...Je crois que je n'avais plus envie de danser. J'étais humiliée par [mon directeur artistique], par toute la danse. Je ne savais plus pourquoi je dansais. Je [ $\left.n^{\prime}\right]$ avais plus qu'une envie, que ça s'arrête.[...]

Renée: Dis ma douleur, serais-tu la danse que je voyais et qui restait figée comme dans mes rêves de petite fille?

La douleur: Il semble que tu commences à me comprendre.

Renée: Attends là c'est moi qui te donne une identité, mais je te demande ton identité.

La douleur: Alors qui je suis vraiment? Je suis la douleur, mais regarde, je ne suis même plus présente comme au début. Si tu me regardes bien, j'ai beaucoup évolué, j'ai changé de place. Tu parles à celle qui n'est même plus là. Je suis juste la douleur, je ne suis pas toi. Je me déplace sans cesse, sans cesse. Je viens et je disparais. Alors je ne sais pas exactement où je suis, mais je ne suis pas toi. $\mathrm{Ou}$ si je suis toi, je bouge avec toi, alors... Mais, non, je ne suis pas toi. Je suis juste une amie, je suis extérieure à toi, Renée. Je suis extérieure à toi et je vais partir quand tu n'auras plus besoin de moi.»

Toutes les blessures, quel que soit leur mode de survenue, ont donné lieu à une douleur d'abord aigue puis s'est chronicisée. Sylvie, par exemple, cherche à tout prix à contourner le problème et, se faisant, retarde sa prise en charge, détériore son équilibre et sa danse. La douleur devenue alors chronique s'insinue ainsi entre les mailles du filet de la discipline 
Douleur Chronique, usages du corps et souci et soi

inhérente au métier. Car le monde de la danse est aussi traversé de lois comme dans un état de droit. Le maître-mot y serait «fonctionner» ou «continuer», comme si de rien n'était. Patricia s'efforce ainsi de terminer sa carrière en beauté, mais c'est alors qu'elle doit se rendre à l'évidence: la douleur a pénétré insidieusement tous les champs de sa vie y compris sa vie de couple. La douleur chronique survient ici et là et limite le sujet dans toutes les activités du quotidien, pas seulement dans son activité professionnelle. «Chaque jour de la douleur, assis sur le divan, difficulté à aller à l'épicerie, faire les achats, parce que ça faisait mal à chaque pas» (Louis). Elle impose sa loi, celle du rien, du vide et de l'immobilité. «Je [ne] pouvais pas attacher mes souliers, je [ne] pouvais pas me plier, il fallait que je me penche complètement le dos droit là comme ça, pour aller attacher mes souliers parce que je [ $n^{\prime}$ ]étais pas capable d'avoir cette flexibilité-là.» (Jeanne)

Ainsi, la douleur chronique ne cesse d'exiger le changement. Chez le danseur professionnel, elle progresse ainsi sur le mode d'une «machine de guerre». Par «machine de guerre», j'entends un processus qui vient questionner les usages du corps dans la danse comme il devrait venir interpeller la pertinence des modèles de représentation et $\mathrm{d}$ 'interprétation à l'oeuvre dans notre société contemporaine. C'est délibérément une autre affaire qui vient clairement poser le problème, plutôt que de l'inscrire dans une théorie et un régime d'énoncés (Deleuze et Guattari, 1980). Le monde de la danse constitue un terrain d'observation privilégié à cet égard, car la douleur chronique vient y interroger les trajectoires habituelles d'un corps perçu comme outil de travail. Elle lui impose ses variations, ses limitations, qui mèneront toutes à des transformations singulières d'un corps qui deviendrait ainsi champ de possible plutôt que champ de bataille. Le monde de la danse constitue à cet égard une sorte de microsociété que la douleur chronique interpelle directement dans son devenir. La machine de guerre douleur chronique s'inscrit dans le temps et y «fait du devenir luimême un modèle» (Deleuze et Guattari, 1980).

Le mode de représentation du corps et de lecture des symptômes occupe une place cruciale dans la résolution de la crise dont témoigne la guérison d'une douleur chronique. D'autres systèmes médicaux, dits holistiques, comme la médecine chinoise, tibétaine ou ayurvédique proposent une vision du corps dans son entièreté et une conception de la santé comme d'un état d'équilibre en renouvellement perpétuel. Elles 
tiennent compte davantage du ressenti et de l'entrecroisement de toutes les lignes d'influence de nature émotionnelle, environnementale ou métabolique. À titre d'exemple, le modèle de représentation de la médecine chinoise considère le corps humain davantage encore comme un paysage avec des vallées, des montagnes et des rivières et en relation directe à l'environnement. (Benoist et Cathebras, 1993). Ce modèle témoigne de l'importance accordée à l'observation de processus connexes qui aboutissent à des accidents, témoins de zones de souffrance ou de turbulence, de manques ou d'excès. La maladie n'y est autre que l'expression d'une zone de souffrance non élucidée (Kar Fung Wu, notes de cours, 2002). La médecine chinoise traditionnelle fonde sa conduite diagnostique et thérapeutique sur le contexte et la réversibilité des situations. Le thérapeute y prend en compte la configuration d'ensemble et recherche les éléments favorables au dénouement des crises sur lesquels prendre appui pour favoriser la guérison. Il est d'ailleurs perçu comme un éveilleur de conscience, quelqu'un qui est capable de stimuler les forces de guérison, de celui ou celle qui souffre et qui détient pourtant toutes les réponses, sans savoir pour autant comment y accéder: «Le propre de la stratégie chinoise selon les anciens traités est de prendre appui sur le potentiel inscrit dans la situation pour se laisser porter par lui au cours de son évolution» (Jullien, 1996: 32). Le rôle du thérapeute y est davantage celui d'un conseiller, voire d'un facilitateur de guérison plutôt que d'un juge ou d'un savant: «Le soin commence par l'acceptation de la réalité phénoménologique de la souffrance du patient, ainsi que le sens que lui donnent le patient et les autres» ${ }^{18}$. La douleur chronique en appelle ainsi d'une révolution en termes de représentations du corps, mais aussi de prise en charge thérapeutique et de prise en compte des dires et des savoirs de celui ou celle qui souffre, par un corps médical qui semble impuissant à résoudre son énigme et s'avère encore trop souvent insensible à une plainte dont le fondement lui échappe. Face à une douleur rebelle, il est clair que chacune de celles qui ont guéri ont fini par exercer leur pouvoir de continuer comme si de rien n'était, ou non. Elles ont ainsi finalement chacune à leur façon réussi à dépasser les lois du métier et de la productivité en modifiant les usages du corps qui déclenchaient une

18 «Caring begins with accepting the phenomenal reality of the patient's suffering, including it's moral significance to the patient and others»(Kirmayer, 1988, p. 82) 
Douleur Chronique, usages du corps et souci et soi

douleur. Pour se faire, il leur a fallu exercer un contre- pouvoir, celui de s'arrêter, de s'écouter et de se remettre en mouvement autrement.

Ainsi, pour accéder à un sens dont le monde médical est privé par trop de contraintes de durée de consultation, d'obligation de résultats et de pratiques basées sur des évidences, il m'aura fallu adopter une posture d'écoute qui légitime absolument les dires de la personne qui souffre et en faire aussi des discours de vérité. Parce qu' «il n'y a pas d'exercice du pouvoir sans une certaine économie des discours de vérité fonctionnant dans, à partir de et à travers ce pouvoir. Nous sommes soumis par le pouvoir à la production de la vérité et nous ne pouvons exercer le pouvoir que par la production de la vérité» (Foucault, 1997, p.22). Ce faisant, je rends compte aussi du pouvoir que chacun et chacune d'entre nous exerce sur son corps et son état de santé. La douleur chronique y serait en définitive une invitation à multiplier et élargir les points de vue, à réintroduire de la chair et du sens dans les dires des patients, à prendre le temps de réintroduire le contexte et l'historique des évènements, bref à se donner les moyens de comprendre et de croire en sa guérison. Et si les patients disaient vrai et que cette insistance, bientôt devenue empêchement puis obstacle incontournable témoignait plutôt des limites d'un «corps perçu comme donné universel et marqueur historique des usages sociétaux en cours» (Mauss, 1934)? La douleur chronique serait alors le reflet d'une société coincée dans un paradigme mécaniste et obnubilée par la productivité, la rentabilité et l'obtention de résultats. En cela elle serait plutôt une sonnette d'alarme et un appel au changement et témoignerait plutôt d'un système vivant et en santé.

\section{Conclusion}

$\mathrm{Au}$ terme de cette analyse force est de constater qu'il n'y a pas de différence de nature ni de sens entre douleur aiguë et douleur chronique. Cette nouvelle catégorie nosographique, dite douleur chronique, ne résiste donc pas à une approche généalogique des usages du corps et disparaît dès lors que l'on sort du cadre qui lui est habituellement réservé dans le monde médical. Ses mécanismes d'installation en sont certes multiples, car tantôt le corps se déforme en cherchant à contourner la blessure initiale, tantôt le verdict médical sème la confusion et induit une dépendance aux 
médicaments ou une défiance en retour. Certes, ces douleurs trouvent parfois leur origine dans un lointain passé ou d'autres fois, dans la répétition à outrance d'un geste inadéquat. Mais, les six récits de guérison tendent à démontrer qu'une douleur chronique n'est jamais qu'une douleur aigue, qui avec le temps se complique ou s'aggrave. La guérison ne peut s'y opérer qu'au prix d'une déconstruction des discours et d'une réorganisation des dynamiques de pouvoir nécessaires à l'élaboration d'une nouvelle posture à la fois physique et mentale. Qu'autant de guérisons surviennent dans une catégorie professionnelle qui échappe aux effets de la catégorisation puisqu'elle rejette d'emblée l'expertise médicale en la matière et ne se fie qu'à sa connaissance du corps, ne peut pas être qu'une coïncidence.

« C'est à l'expérience fondamentale entre choix conscient et accident et à leurs conséquences morales que la pratique médicale devrait s'intéresser si elle veut pouvoir répondre de façon sensible au besoin émotionnels des patients et aux implications sociales de la détresse» (Kirmayer, 1988, p.58) ${ }^{19}$.

En effet, les trajectoires de guérison décrédibilisent un savoir médical basé sur des évidences et qui se fie aveuglément aux «guidelines». Qui plus est, en qualifiant la douleur de «rebelle au traitement» ou de «chronique», le monde médical met les patients au défi de guérir car une composante aussi essentielle qu'évidente de la guérison est bien d'y croire. La catégorisation d'une douleur en douleur chronique semble donc tout à fait contre-productive à cet égard. Elle suffit à elle seule à justifier que bien des personnes s'installent dans un état de fait ou soient tentées de tirer profit d'une condition faussement jugée irréversible. Et dans tous les cas, si rébellion il y a dans cette douleur qui persiste au-delà de normes jugées acceptables, elle participe pleinement de la guérison.

En resituant ainsi la «maladie» dans une temporalité qui lui est

19 «It is the fundamental experiences of agency and accident, and their moral consequences, that must be adressed if medical practice is to respond sensitively to the emotional needs of patients and the social implications of distress» 
Douleur Chronique, usages du corps et souci et soi

propre, il est possible de déconstruire cette catégorie et de redonner ses lettres de noblesse à un processus complexe aux multiples entrées et pourtant toujours singulier. Mais, parce que «nous avons tous du pouvoir dans le corps» (Foucault, 1997: 27) et que le pouvoir prend appui sur des besoins, parce que le droit à la santé est reconnu comme un droit universel, la question du pouvoir dans le domaine de la santé n'a pas de réponse simple. Dans le cas particulier des professionnels de la danse, la douleur chronique peut ainsi représenter un frein à la volonté de briller, de se produire sur scène, de performer à tout prix, de se conformer aux dictats de la société du spectacle. Se faisant, la douleur chronique vient enrayer la machine de la production culturelle et grossir la masse des handicaps. Elle met aussi de l'avant un corps sensible et du vécu. Elle oppose symptômes subjectifs et objectifs et contribue à poser le problème des usages du corps quand le besoin de conformité au modèle sociétal dominant rend impératif de fonctionner à tout prix. Parce que la douleur chronique signale définitivement un problème en lien avec les usages du corps qui, avec le temps, mène à l'immobilisation, elle fait ainsi office de grain de sable dans la machine économique comme dans la machine physique. Elle vient empêcher de faire comme si de rien n'était et même si l'institution médicale tend à la réduire au silence, en prescrivant des antalgiques de plus en plus apparentés à des anesthésiques, le problème sous-jacent continue son œuvre d'obstruction et vient alourdir la facture des indemnités de salaire et des traitements.

La douleur chronique en appelle d'une sortie de crise et d'une profonde mutation de nos modes de représentation du corps et de ce qui fait sens. Elle souligne l'inadéquation des modèles mécaniste ou duel opposant le corps à l'esprit, le visible à l'invisible, le sensible au rationnel et l'endurance à la douleur. Elle exige d'échanger un regard incisif, séparateur et scrutateur (Foucault, 1972) pour un regard englobant, myope voire aveugle, non jugeant et à l'écoute, garant d'une conduite thérapeutique intuitive.

La douleur chronique n'est finalement que le symptôme d'une crise sociétale doublée d'une crise du sujet dont la lecture est facilitée par l'échappement du monde de la danse au monde médical et aux savoirs experts: «Un corps en santé fournit un modèle de plénitude organique, un corps malade en fournit un de désaccord social, de conflit et de désintégration. Réciproquement, une société malade ou en santé offre un 
modèle de compréhension du corps». ${ }^{20}$ De la même façon, en immobilisant le corps social sous le poids du fardeau des indemnités de salaire et des remboursements de médicaments, la douleur chronique en appelle d'un changement de paradigme dans la compréhension et dans la réponse apportée aux symptômes dits subjectifs tant chez les médecins que chez les employeurs et tous les intermédiaires. Car dans tous les cas de guérison, la sortie de crise a nécessité une mise au repos et un réajustement des gestes et des usages du corps, une grande transformation et une résolution de conflit entre le corps et l'esprit qui suggère un changement de culture qui substitue la souplesse à l'endurance, la qualité à la quantité. Le monde de la danse indique ainsi de multiples issues heureuses dont le plus petit dénominateur commun serait le changement des usages du corps: arrêter de faire ce qui fait mal, changer de carrière ou se donner les moyens de choisir son environnement de travail.

Par ailleurs puisque toutes les lésions auxquelles les interprètes font allusion sont d'ordre musculo-squelettique, il est raisonnable de penser appliquer les résultats de cette analyse à toutes les douleurs en lien avec les usages du corps. Quels que soient les corps de métier, le corps y est soumis inéluctablement à des rapports de forces similaires, d'ordre émotionnel et existentiel aussi bien que professionnel et thérapeutique. Les qualités d'endurance retrouvées au travers de tous les récits permettent également de suggérer que les données s'appliquent indifféremment à toutes les professions y compris celles qui réclament une grande résistance à l'effort physique, comme le métier de manutentionnaire, de chauffeur de poids lourd ou d'autobus. De la même façon, ces données s'appliquent aux postes plus sédentaires, mais tout aussi exposés au stress et aux mauvaises conditions de travail y compris d'ordre ergonomique ou aux autres métiers de vocation exposés à une culture de l'endurance.

\section{Références}

20 «The body in health offers a model of organic wholeness; the body in sickness offers a model of social disharmony, conflict, and disintegration. Reciprocally, society in «sickness» and in «health» offers a model for understanding the body»(Scheper- Hugues et Lock 1987: 7). 
Douleur Chronique, usages du corps et souci et soi

AGAMBEN G., Qu'est-ce qu'un dispositif ?, Petite bibliothèque Rivage, 2007. ALLUÉ M., La douleur en direct. Anthropologie et Sociétés, vol. 23, n² 2, 1999, p.117- 137.

BARIL R. et al., TMS, l'influence des représentations de la maladie, de la douleur et de la guérison sur le processus de réadaptation au travail. Rapport R 544, IRSST, 2008.

BARRAS H., De l'art cuit à l'art cru, aux sources de la création, éd. Liber, coll. Les Impatients, 2007.

BASTIN C., La plainte du corps. Revue Danser, mars 2010.

BASZANGER I. Émergence d'un groupe professionnel et travail de légitimation. Le cas des médecins de la douleur. Revue française de sociologie, vol. 31, No 2, 1990, p.257- 282.

BECKER B. Narratives of pain in later life and conventions of storytelling. Journal of aging studies, vol.13, No1, 1999, p.73-87.

BENOIST J. et Cathebras P. Conceptions et représentations du corps. Les classiques des sciences sociales, http://classiques.uqac.ca/, 1993

COUTU MF, Baril R, Durand MJ, Cote D, Rouleau A, Cadieux G. Transforming the meaning of PAIN: an important step for the return to work. Work, No 35, 2010, p.209-219.

DELEUZE G. et Guattari F., Mille Plateaux, Capitalisme et schizophrénie 2, éd. de minuit, Paris, 1980.

DELEUZE G., Qu'est-ce qu'un dispositif ? Michel Foucault philosophe, rencontre internationale, Paris 9-11 janvier 1988 . Des travaux. éd. Seuil, 1989, p.185-195.

DROIT R-P., Michel Foucault: entretiens, éd Odile Jacob, Paris, 2004.

DURAND R. Que le théâtre et l'avenir de la danse ou réciproquement. Art Press, 1988, p. 34-36.

EISENBERG L. Disease and illness. Culture, Medicine and Psychiatry 1, 1977, p. 9-23.

FOUCAULT M., Naissance de la clinique, une archéologie du regard médical, éd. Presses Universitaires de France, Paris, 1963.

. Surveiller et punir. Naissance de la prison, éd. Hachette, Paris, 1975.

. Il faut défendre la société. Cours au collège de France, 1976, éd. du Seuil/ Gallimard, Paris, 1997.

Sécurité, territoire, population, Cours au collège de France 
1977-1978, éd. du Seuil/Gallimard, Paris, 2004.

- Naissance de la biopolitique, Cours au collège de France 1978-1979, éd. du Seuil/Gallimard, Paris, 2004.

. l'Herméneutique du sujet, Cours au collège de France, 19811982, éd. du Seuil/Gallimard, Paris, 2001.

GINOT I. et LAUNAY I. L'école une fabrique d'anticorps. Art Press, hors série avril 2002, p.106-111.

HALIOUA B., 2009, Histoire de la médecine, éd. Masson, Paris.

IZRINE A., La danse dans tous ses états, éd. L'Arche, Paris, 2002.

JACKSON J.E. Stigma, liminality and chronic pain, mind-body borderlands. American ethnologist, vol.32, No 3, 2005, p.332-353.

JULLIEN F., Traité de l'efficacité, éd. Grasset, Paris, 1996.

KIRMAYER L. Mind and body as metaphors: hidden values in biomedicine. in: M. Lock \& D.R. GORDON (eds). Biomedicine examined. Dordrecht, Kluwer Academic Publishers, 1988, p. 57-96.

KLEINMAN A. et al. Pain as human experience: an introduction, in Pain as Human Experience: An Anthropological Perspective, edited by Del VecchioGood M-J.et al., University of California Press, Berkeley and Los Angeles, California, 1992.

LOUPPE L., éditorial, «Les années danse», Art Press, special No 8, 1988, p.3. LOUPPE L., «L'éveil du corps», Art Press, special No 8, 1988, p.12.

LOUPPE L., "Cunningham, maintenant et toujours», Art Press, special No 8, 1988, p.6-8.

MARANDA M.F. et al., La détresse des médecins: un appel au changement. éd. Presses de l'Université Laval, Québec, 2006.

MASSÉ R., «Les conditions d'une anthropologie sémiotique de la détresse psychologique», Recherche sémiotique, vol.19, No 1, 1999, p. 39- 62.

MAUSS M., «Les techniques du corps», édition électronique de Sociologie et anthropologie, réalisée PAR Jean-Marie Tremblay, article originalement publié dans le Journal de Psychologie, vol. XXXII, No 3-4, 1936, Communication présentée à la Société de Psychologie le 17 mai 1934.

PERREAULT M., «Les blessures et leur prévention chez les danseurs professionnels», l'IRSST, avril 1988.

REY R. Histoire de la douleur. éd. la Découverte, Paris, 1993.

ROGOZINSKI J. Guérir la vie, Passages. éd. du Cerf, Paris, 2011. 
Douleur Chronique, usages du corps et souci et soi

SCHEPER-HUGUES N. and Lock M., «The Mindful Body: A Prolegomenon to Future Work in Medical Anthropology», Medical Anthropology Quarterly, vol.1, No1, 1987, p.6-41.

SCOTT J.C. Weapons of the Weak: Everyday Forms of Peasant Resistance. Yale University Press, 1985.

SORIGNET P.E., «Sortir d'un métier de vocation: le cas des danseurs contemporains», Sociétés contemporaines, No56, 2004, p111-132.

SUDDATH C., «Living with pain; what happens when you can't make it go away», Time, vol 177, N.9, 2011.

TEMBEK I. Pour une culture chorégraphique élargie. Bilan inhabituel de la danse québécoise. traité de la culture, publié sous la direction de Denise Lemieux; avec la collaboration de Gilles Bibeau, édition IQRC, Presses de l'Université Laval, Québec, 2002, p.769-786.

TURNER B. S. The body and society. Explorations in social theory. Basil Blackwell Ltd, London, 1984. 